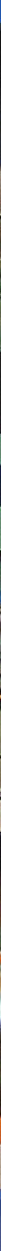

Kongresshaus, Biel, 28. Mai 2009

\title{
Protokoll der ordentlichen Ärztekammer
}

Erika Flückiger,

Leiterin Dienste

Ein Glossar mit den am häufigsten verwendeten Abkürzungen finden Sie auf Seite 1112.

(Anmerkung der Protokollführerin: Zum besseren Verständnis werden die Geschäfte in der Reihenfolge der eingangs der Sitzung geänderten Traktandenliste protokolliert.)

\section{Begrüssung, Mitteilungen, Bestellung des Büros}

Nach Erreichen des erforderlichen Quorums der Hälfte der stimmberechtigten Delegierten eröffnet der Präsident, Jacques de Haller, um 9.35 Uhr die ordentliche Sitzung der Ärztekammer. Er begrüsst die Anwesenden und die Gäste: Kurt Schreier, designierter Präsident der Ärztegesellschaft des Kantons Schwyz, Reinhard Kunz, Geschäftsführer der FMH Services, Peter Geissbühler, von Graffenried Treuhand AG (Kontrollstelle) für Traktandum 3, die Mitglieder der Geschäftsprüfungskommission (GPK), Roland Schwarz, Philippe Rheiner und Adrian Sury, sowie Lukas Golder, gfs.bern, für Traktandum 5. Im Anschluss an einige administrative und organisatorische Informationen überbringt der Präsident der Ärztekammer die traurige Nachricht über den plötzlichen Hinschied von François Bossard, Hilterfingen, der am Vorabend an einem Herzinfarkt verstorben ist. Jacques de Haller würdigt in kurzen Worten den Verstorbenen, der sich während Jahrzehnten äusserst aktiv in der Standespolitik, insbesondere in Tariffragen, eingesetzt und sich grosse Verdienste erworben hat. Der Präsident drückt der Gattin und der Familie von François
Bossard sein tiefes Beleid aus. Die Anwesenden erheben sich zum Gedenken an den Verstorbenen.

Anschliessend bestellt der Präsident das Büro der heutigen Ärztekammersitzung, welchem der Präsident, die beiden Vizepräsidenten (Ignazio Cassis wird wegen der Sitzung des Nationalrates erst am Nachmittag eintreffen), der Generalsekretär und die folgenden Stimmenzähler angehören: Franziska Zogg/ZG, Christoph Ramstein/VEDAG, Alain Souche/GE, Marcel Stampfli/BE, Christian Kind/SGP, Chantal Bonnard/SGH, Michael Canonica/ZH, Anton Gehler/SGIM, Stefan Schumacher/ VSAO, Lars Frauchiger/VSAO.

Den Zeitpunkt, nach welchem keine Abstimmungen und Wahlen mehr stattfinden können, legt der Präsident auf $17.00 \mathrm{Uhr}$ fest.

\section{Traktandenliste}

Nach Ablauf der Frist für die Einreichung von zusätzlichen Traktanden (Anm. der Protokollführerin: bis spätestens 5 Wochen vor der Sitzung) sind eine Reihe von Anträgen auf Traktandierung eingetroffen. Die ordentlichen Delegierten haben in einem Nachversand per Mail (22. Mai 2009) die bis zu diesem Zeitpunkt 
eingetroffenen Anträge sowie die provisorisch angepasste Traktandenliste erhalten. Ein weiterer Antrag auf Nachtraktandierung (Traktandum 7.6.) ist zu Beginn der Sitzung von Christoph Bosshard/VSAO eingereicht worden.

Über Nachtraktandierungen muss die Ärztekammer mit 2/3 Mehr beschliessen. Da nach Erachten des Präsidenten alle Nachtraktantierungs-Anträge unbestritten sind, schlägt er der Ärztekammer vor, global über deren Aufnahme in die Traktandenliste abzustimmen. Es gibt keine Einwände gegen diesen Vorschlag, und die Ärztekammer beschliesst mit $2 / 3$ Mehr global für alle eingereichten Anträge die Aufnahme in die Traktandenliste.

Es liegen weiter drei Anträge auf Umstellung der Traktandenliste vor: Antrag Nr. 1 zu Traktandum 1.1., Jürg Schlup/BE: Traktandum 17 soll neu Traktandum 8 werden; Antrag Nr. 2 zu Traktandum 1.1., Jean-Pierre Pavillon/VD: Traktandum 17.2. soll neu Traktandum 5 werden; Antrag Nr. 3 zu Traktandum 1.1., Christoph Ramstein/VEDAG: Traktandum 18 soll neu Traktandum 8 werden. Den Antragstellern geht es vor allem darum, die wichtigen Informationen aus den Ressorts «Tarife und Verträge» und «Medical Education» nicht erst am Ende des Tages zu behandeln, wenn sich die Reihen der Delegierten erfahrungsgemäss bereits gelichtet haben. Um diesen Anliegen Rechnung zu tragen, schlägt der Präsident vor, im Laufe des Tages die Informationen aus den Ressorts zwischen den in der Traktandenliste aufgeführten Traktanden einfliessen zu lassen. Die Informationen aus dem Ressort «Tarife und Verträge» würden so noch vor dem Mittagessen an die Reihe kommen, diejenigen des Ressorts «Medi-

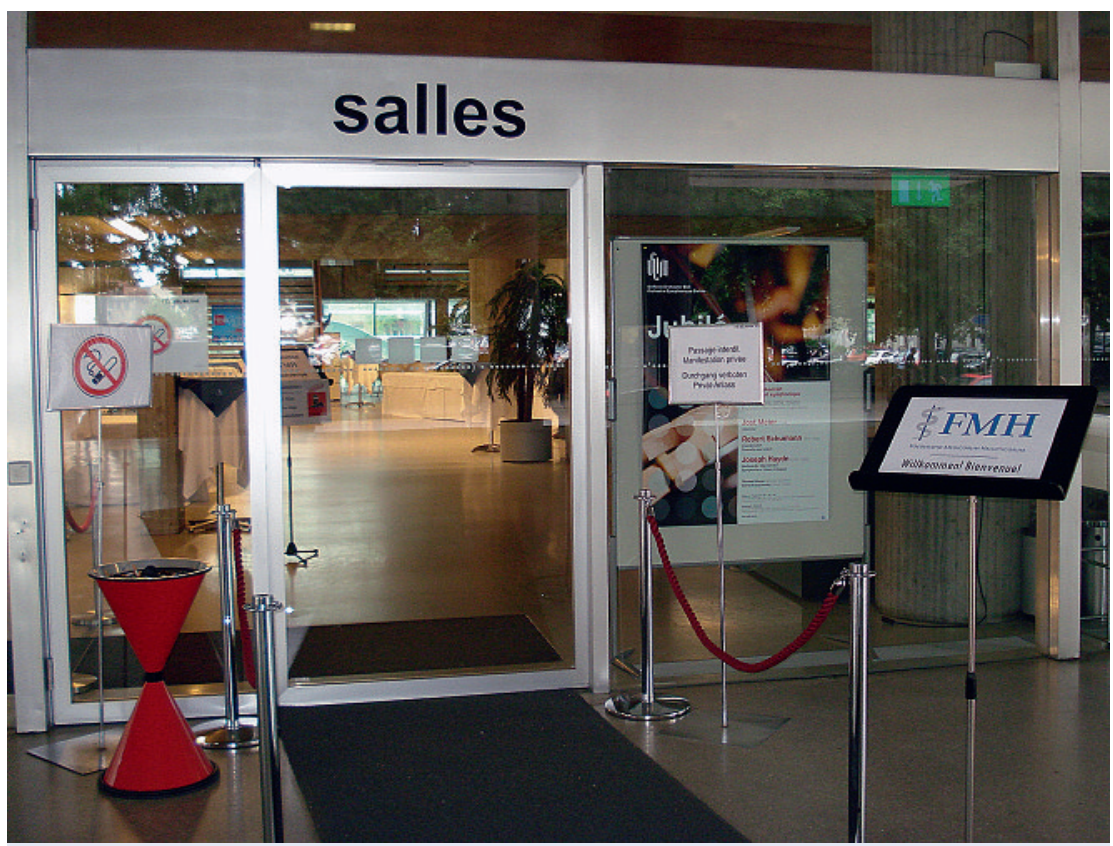

Alles bereit: Türöffnung für die Ärztekammer-Delegierten. cal Education» am frühen Nachmittag. Alle betroffenen Antragsteller erklären sich mit diesem Vorgehen einverstanden. Die Traktandenliste gilt somit als genehmigt.

Der Vollständigkeit halber werden die nachträglich aufgenommen Traktanden hier aufgeführt: Traktandum 7.2: GPK-Amtszeitverlängerung, Antrag Roland Schwarz/GPK; Traktandum 7.3.: Sitzverteilung ÄK, Antrag Hans Kurt/SGPP; Traktandum 7.4.: Fähigkeitsausweis und FMH-Beitrittspflicht, Antrag Fabrice Dami/ VSAO; Traktandum 7.5.: Liste der ÄK-Delegierten, Antrag Jean-Pierre Pavillon/VD; Traktandum 7.6.: Gastrecht für VSAO im ZV FMH, Antrag Christoph Bosshard/VSAO; Traktandum 10.: zusätzliche SAMW-Richtlinie (Patientenverfügungen), Antrag ZV.

Der Präsident erteilt das Wort anschliessend dem Generalsekretär für die Präsentation der neuen Mitarbeiter/innen des Generalsekretariats. Daniel Herzog informiert die Ärztekammer über folgende Personal-Neuzugänge seit der a.o. Ärztekammer vom 11.12.2008: Sébastian Lerch/Gutachterstelle, Eintritt 1.1.2009; Lea Schläpfer/DDQ (Daten, Demographie, Qualität), Eintritt 1.2.2009; Petra Ingenpass/Tarifdienst als Stv. Leiterin SwissDRG, Eintritt 1.4.2009; Erika Fankhauser/ Vorstandssekretariat, als neue Teamleiterin, Eintritt 1.6.2009.

\section{Jahresbericht des Zentralvorstandes und der Geschäftsprüfungskommission: Berichtsperiode 2008}

Der Präsident verweist auf den Geschäftsbericht 2008, welcher am 13. Mai 2009 mit der Nr. 20 der Schweizerischen Ärztezeitung (SÄZ) allen FMH-Mitgliedern zugestellt worden ist. Roland Schwarz/Präsident GPK greift aus dem Jahresbericht der GPK, welchen die Delegierten mit der Einladung zur heutigen Sitzung erhalten haben, vier Punkte heraus:

Punkt 1: Die Anstellungsverhältnisse der Mitglieder des Zentralvorstands (ZV) sind neu geregelt worden. Bei den bisherigen Mandatsverträgen bestand ein erhöhtes Risiko für die Bereiche AHV und BVG, weil die Steuerbehörden Arbeitspensen über 30\% nicht mehr als Nebenbeschäftigung akzeptierten, was Nachzahlungen durch die FMH und einzelne ZV-Mitglieder zur Folge hätte. Deshalb wurden sämtliche bisherigen Mandatsverträge kostenneutral in Teilzeitarbeitsverträge umgeändert und die Bereiche AHV und BVG neu geregelt.

Punkt 2: Die GPK hat festgestellt, dass bei den Klausursitzungen des ZV die Vorschriften der Geschäftsordnung (GO) nicht immer eingehalten worden sind. Auch bei formlosen Zusammenkünften eines Teils oder des ganzen ZV ist die GO immer dann einzuhalten, wenn Beschlüsse gefasst werden. Diese sind zusammen mit den ihnen vorangegangenen Hauptüberlegungen zu protokollieren und dem in der GO vorgesehenen Empfängerkreis - dazu gehört auch die GPK - zuzustellen. 
Punkt 3: In Sachen Budgetstabilisierungsmassnahmen (ÄK-Beschluss 2007) sind Modul 1/keine Rückerstattung mehr von Mitgliederbeiträgen nach Titelerwerb ab 1.1.2007 und Modul 2/Rückerstattung von Mitgliederbeiträgen bei Titelerwerb, kompensiert mit Beitrag ab 1.1.2008, umgesetzt worden. Bei Modul 3 konnte bei den Betriebsaufwendungen des ZV im Jahr 2008 eine Aufwandreduktion von $-7 \%$ gegenüber dem Vorjahr erreicht werden. Modul 4/Restrukturierung Generalsekretariat wurde soweit umgesetzt, als die beiden Gutachterstellen zusammengelegt wurden, mit Sitz in Bern, und beim Übersetzungsdienst ein TeilOutsourcing erfolgte. Damit erachtet die GPK diese Massnahme als umgesetzt.

Punkt 4: Das Interne Kontrollsystem/IKS, welchem die FMH nach Gesetz unterliegt, wurde mit grossem Einsatz und externer Mithilfe eingeführt. Die GPK hat sich vergewissert, dass die Vorgaben genau umgesetzt wurden. Es wird in den kommenden Jahren zu prüfen sein, ob die getroffenen Massnahmen zweckmässig und vom Aufwand her vertretbar sind. Der Präsident wird den Jahresbericht 2008, die Jahresrechnung 2008 und die Décharge-Erteilung am Schluss des Traktandums 4 der Ärztekammer zur Abstimmung unterbreiten.

\section{Jahresrechnung 2008 der FMH}

Sie wird vom Generalsekretär, Daniel Herzog, präsentiert, der zu Beginn auf die verschiedenen Beilagen hinweist, welche den Ärztekammerdelegierten zugestellt wurden: Jahresrechnung 2008 der FMH mit Bericht der Kontrollstelle, Bilanz, Erfolgsrechnung und Anhang, Bemerkungen zur Jahresrechnung, Bericht der GPK, Erfolgsrechnung des Sekretariates Aus-, Weiter- und Fortbildung (AWF), Jahresrechnung des Hilfsfonds der FMH und Jahresrechnung zur Förderung der Weiterbildung von Schweizer Ärzten in Entwicklungsländern.

Es wurde ein Gewinn von CHF 696955.03 erwirtschaftet (2007: CHF 352474.00). Das Budget 2008 hatte einen Verlust von CHF -250457.00 vorgesehen. Der Mitgliedergrundbeitrag wurde wieder auf CHF 530.00 gesenkt (Vorjahr CHF 630.00). Die Ursache für den Gewinn liegt mehrheitlich bei den Einnahmen der AWF. An Rückerstattungen bei/nach Titelerwerb wurden CHF 1577267.00 ausbezahlt. Damit ist die Spitze erreicht, und die Rückerstattungen werden in den nächsten Jahren rückläufig ausfallen. Aus dem Gewinn werden den AWF-Reserven CHF 893000.00 zugewiesen, wodurch der FMH selbst ein Verlust von CHF -196082.00 verbleibt.

Ch. Ramstein/VEDAG hat zwei Fragen zur Jahresrechnung 2008: Die erste betrifft den Verlag EMH, welcher u. a. das offizielle Organ der FMH, die Schweizerische Ärztezeitung (SÄZ), herausgibt. Wie derzeit die meisten Zeitschriften, hat auch EMH mit rückläufigen Inseraten zu kämpfen. Ch. Ramstein möchte wissen, ob der Zentralvorstand Massnahmen ergriffen hat, um EMH in die Zukunft hinüberzuretten. Der Präsident versichert, dass der Zentralvorstand in enger Diskus- sion mit dem Verwaltungsrat EMH steht. Eine der bereits getroffenen Massnahmen ist die Reduktion der Lizenz, welche die EMH an die FMH bezahlt. Sie wurde um die Hälfte, auf CHF 500000.00, reduziert. Auch die Anzahl der Gratisabonnemente wurde beträchtlich reduziert. Demgegenüber hat sich der ZV gegen die von EMH einseitig getroffene Massnahme, darin bestehend, die SÄZ den im Ausland lebenden FMH-Mitgliedern nicht mehr gratis zu liefern, zur Wehr gesetzt: Jedes FMH-Mitglied soll die SÄZ gratis erhalten! In Prüfung sind zurzeit eine ganze Reihe weiterer Massnahmen. Der Fragesteller bedankt sich für die Antwort. Auf seine zweite Frage, wo die Aufwendungen für die PR-Agentur Furrer.Hugi \& Partner AG verbucht seien, verweist der Generalsekretär auf das Konto «Werbeaufwand» und die dazu gehörende Bemerkung Nr. 29: Mit Furrer.Hugi wurden eine professionelle Medienbeobachtung und ein Monitoring aufgebaut, der Aufwand durch Auflösung von Rückstellungen (PR und Politik) abgebucht. Der Fragesteller zeigt sich auch von dieser Antwort befriedigt.

Der Präsident übergibt das Wort anschliessend an R. Schwarz/GPK und P. Geissbühler/Kontrollstelle. Roland Schwarz/Präsident GPK orientiert, dass die GPK an der Besprechung mit der Kontrollstelle teilgenommen hat und die Ordnungsmässigkeit der Jahresrechnung habe feststellen können. Gemäss $R$. Schwarz kann das (für die FMH) ausgewiesene Defizit sehr gut verkraftet werden. Die GPK empfiehlt deshalb der Ärztekammer Annahme der Jahresrechnung und Déchargeerteilung an den Zentralvorstand. Auch Peter Geissbühler/Kontrollstelle von Graffenried Treuhand AG, empfiehlt die Jahresrechnung zur Annahme, indem er auf den schriftlichen Revisionsbericht in den Unterlagen zum vorliegenden Traktandum hinweist. Aus dem Plenum werden keine weiteren Fragen gestellt.

\section{Décharge-Erteilung an den FMH-Zentralvorstand}

Der Präsident bittet deshalb Ch. Ramstein, seinen Antrag Nr. 2 zu Traktandum 4 zu erläutern. Christoph Ramstein/VEDAG hatte beantragt, dem Zentralvorstand für das Geschäftsjahr 2008 nur dann Décharge zu erteilen, wenn ab 2009 in den Geschäftsberichten (erstmals im Geschäftsbericht 2009) die Entlöhnung transparent aufgeführt wird. Der Antragsteller will nun seinen Antrag nicht mehr mit der Décharge-Erteilung verknüpfen, plädiert aber für die künftige Transparenz bezüglich der Entlöhnungen, denn die Basis habe ein Anliegen zu wissen, was mit den Mitgliederbeiträgen geschehe. Da der Antrag Nr. 2 zu Traktandum 4 nun keine Verknüpfung mehr mit der Décharge-Erteilung enthält, wird ihn der Präsident anschliessend an die Abstimmungen zur Diskussion bringen.

Nachdem keine Wortmeldung mehr gewünscht wird, unterbreitet der Präsident die folgenden Anträge der Ärztekammer zur Genehmigung:

Antrag Nr. 1 zu Traktandum 2/Jahresbericht des ZV und der GPK: Der Antrag wird mit überwältigender 


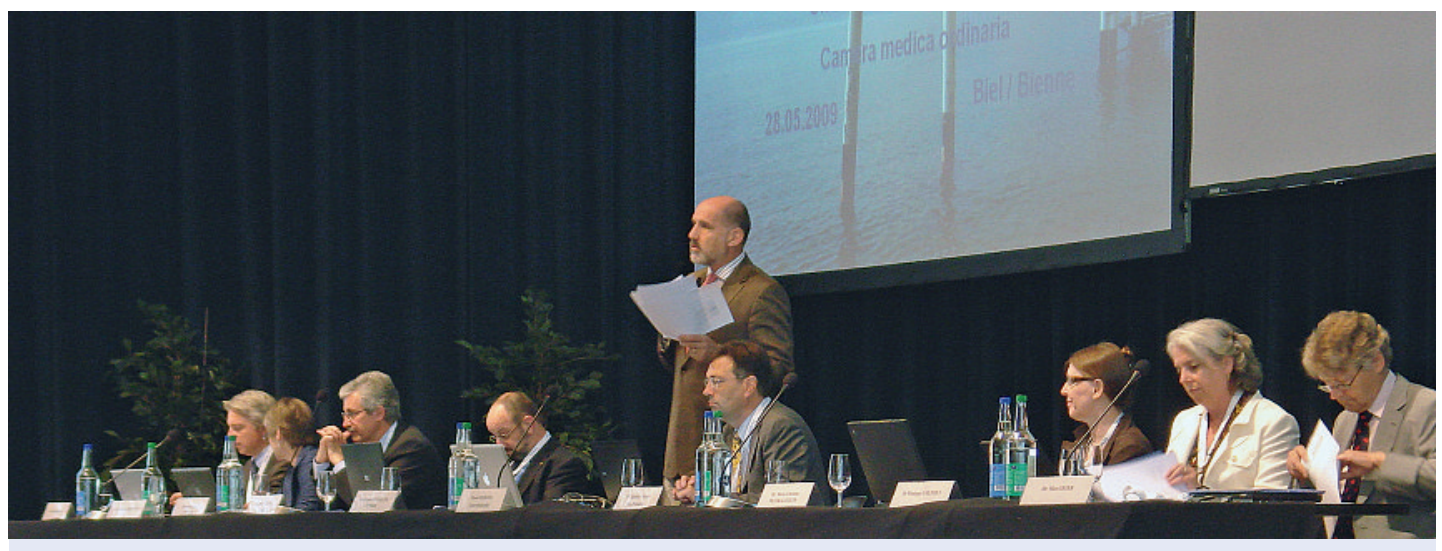

FMH-Präsident Jacques de Haller eröffnet die Ärztekammer.

Mehrheit, mit 1 Gegenstimme und ohne Enthaltungen, angenommen.

Antrag Nr. 1 zu Traktandum 3/Jahresrechnung 2008 der FMH: Der Antrag wird ohne Gegenstimmen und bei 2 Enthaltungen angenommen.

Antrag Nr. $1 \mathrm{zu}$ Traktandum 4/Décharge-Erteilung an den FMH-Zentralvorstand: Auch dieser Antrag wird ohne Gegenstimmen und bei 2 Enthaltungen angenommen.

Der Präsident stellt wie versprochen nun den $A n$ trag Nr. 2 zu Traktandum 4 von Christoph Ramstein/ VEDAG zur Diskussion. Er erwähnt einleitend, dass er bei Journalistenfragen nach Salären bei der FMH immer sehr zurückhaltend sei, insbesondere deshalb, weil es dabei immer zu Missverständnissen komme. Aus diesem Grund möchte er auch die Saläre der ZVMitglieder nicht öffentlich machen, was aber keineswegs als Statement gegen Transparenz zu verstehen sei. Christine Romann/ZV steht dafür ein, dass die ZVSaläre öffentlich gemacht werden. Pierre-Alain Schneider/GE plädiert für Zurückhaltung gegenüber der Öffentlichkeit, aber für transparente Information der Delegiertenversammlung (DV). Für Thomas Heuberger/ $B E$ stellt der Antrag ein klassisches Postulat dar, dessen Aufnahme in die GO er im Verlauf der heutigen Sitzung noch beantragen wird. Der ZV solle zunächst darüber entscheiden, wo man die Saläre publik machen will. Die Ärztekammer solle heute dazu keinen Beschluss fassen. Christoph Ramstein/VEDAG lässt sich von seinem Vorredner überzeugen und zieht seinen Antrag zu Gunsten des Vorschlags von Th. Heuberger zurück. Der Präsident nimmt den Antrag zuhanden des Zentralvorstands entgegen.

(An dieser Stelle werden die Informationen aus dem Ressort DDQ eingeschoben: s. Traktandum 14.)

\section{Politische und strategische Zielsetzungen/ Jahresziele FMH; Mitgliederbefragung}

Der Präsident gliedert seine Präsentation in 3 Teile: Jahresziele 2008-2012/Umfrage bei Mitgliedern/Denkanstösse, einige Statements zur Zukunft.
Jahresziele 2008-2012

Sie wurden von der a.o. Ärztekammer im Dezember 2008 genehmigt, anschliessend auf der FMH-Website und in der SÄZ publiziert. In den darauf folgenden Monaten hat sich der ZV mit der Entwicklung einer Umsetzungsplanung beschäftigt, die dazu dienen soll, die Arbeiten thematisch und zeitlich zu priorisieren und innerhalb des ZV einem Ressort bzw. einer verantwortlichen Person zuzuweisen. Konkret wurden zu jedem Ziel Eckwerte erarbeitet, die Priorität festgelegt, Massnahmen und der entsprechende Zeitplan, innerhalb dessen diese umzusetzen sind, sowie die Zuständigkeit festgehalten. Im Sommer wird sich der ZV mit der Budgetrelevanz und den möglichen Auswirkungen befassen, welche diese auf gewisse Abteilungsbudgets haben werden, sei es, dass die Aufgaben nicht vorhersehbar gewesen sind, sei es, dass die Prioritäten anders zu setzen sind. Der Präsident zeigt an einigen konkreten Beispielen auf, wie sich das Strategiepapier mit den geschilderten Ergänzungen nun präsentiert.

Parallel dazu wurde eine Mitgliederbefragung durchgeführt, deren Resultate in die einzelnen Zielsetzungen einzuarbeiten sind. Dieser Prozess ist noch nicht abgeschlossen, weshalb es keinen Sinn macht, bereits heute Jahresziele vorzuschlagen.

Mit der ordentlichen Ärztekammer 2010 wird man aber zum normalen, statutarisch vorgesehenen Rhythmus zurückkehren, ein aktualisiertes Papier vorlegen und die Jahresziele diskutieren und festlegen lassen.

\section{Mitgliederbefragung}

Der Präsident erinnert an die Zielsetzung 7.1. im Strategiepapier, die festhält: «Die FMH kennt und berücksichtigt die Bedürfnisse und Anliegen ihrer Mitglieder.» Der ZV hat deshalb Offerten eingeholt für die Durchführung einer Mitgliederbefragung und den Auftrag schliesslich an die gfs.bern vergeben. Er begrüsst Lukas Golder, gfs.bern, und bittet ihn, die Resultate dieser Umfrage zu präsentieren.

Lukas Golder ist sich bewusst, dass die Anwesenden von ihm heute nicht nur eine Analyse, sondern auch 
eine Diagnose erwarten. Wäre er Mediziner und Lateiner, würde er aufgrund der Umfrageresultate die Diagnose «lleus magnus» stellen, also eine Blockade. Mit andern Worten kann man sagen, dass die FMH sich, wenn auch noch ungenügend, auf dem Weg von einer Standesorganisation hin zu einer politischen Bewegungsorganisation befindet. Eine politverdrossene Ärzteschaft gewichtet die politische Einflussnahme als sehr hoch. Er informiert anschliessend kurz über das technische Vorgehen bei der Befragung, die zwischen dem 16. Februar und dem 7. März 2009 stattgefunden hat. Sie wurde online durchgeführt, allerdings für gewisse Gruppen mit einer telefonischen Befragung ergänzt. Sämtliche FMH-Mitglieder wurden mindestens einmal kontaktiert, wer keine E-Mail-Adresse hat, erhielt einen Brief. Schliesslich haben 7897 Ärztinnen und Ärzte teilgenommen, das sind 23\% aller Mitglieder, was ein ausserordentlich hoher und guter Rücklauf ist. Der Rücklauf ergab auch eine fast perfekte Verteilung nach Kantonen und Geschlecht. Auf die Resultate wird an dieser Stelle nicht näher eingegangen. Die detaillierten Umfrageergebnisse zu den einzelnen Fragen sind ab dem 1. Juni auf der FMH-Website zugänglich und werden am 10. Juni in der SÄZ publiziert. Es sei hier nur noch auf die Synthese der gfs.bern hingewiesen, die für die kommenden Diskussionen innerhalb des Verbandes 4 Arbeitsthesen formuliert:

Arbeitsthese 1: Die Haltung zu den strategischen Zielen und zur Ausrichtung der FMH.

Die politischen Entwicklungen seit dem KVG haben die Ärzteschaft erheblich politisiert und frustriert. Die politische Tätigkeit der FMH hinkt dieser Entwicklung nach, und es besteht Verbesserungsbedarf.

Arbeitsthese 2: Die Bedürfnisse und die berufspolitischen Interessen.

Zwar gibt es einen erheblichen Teil der Ärzteschaft, für welchen nur der FMH-Titel Priorität hat; die Information und die Ausbildung behalten auch ihre Bedeutung im Rahmen dieser Kerninteressen. Trotzdem verlagern sich die Bedürfnisse klar hin zu einer Intensivierung der Interessenpolitik im Gesundheitswesen und zu mehr politischer Einflussnahme.

Arbeitsthese 3: Gemeinsame und breit akzeptierte Basis des Handelns.

Die FMH erfüllt das Soll bei den klassischen Leistungen und allen voran bei der Ausbildung. Die aktuelle Führung und die Kommunikation werden relativ gut bewertet. Es besteht eine solide Basis, um die politische Interessenvertretung $\mathrm{zu}$ intensivieren. Diese wird polarisiert und im Schnitt ungenügend bewertet. Gleichzeitig bestehen namentlich im Bereich eHealth weitere Optionen, um die Dienstleistungen als Standesorganisation auszubauen

Arbeitsthese 4: Einstellungen gegenüber der FMH, Leistungsbeurteilung und die Erwartungen an den Verband.

Die Standesorganisation sollte mindestens zu einem Teil in eine Bewegungsorganisation umgewandelt werden. Kernelemente davon sind professionelle Kampagnenführung und ein professionelles Lobbying.
Aus dem Plenum werden anschliessend Fragen nach weiteren gezielten Auswertungen gestellt. Der Präsident bestätigt, dass die Daten zur Verfügung stehen und Interessengruppen weitere Auswertungen bei der gfs.bern verlangen können. Die Präsentation stellt so etwas wie eine aktuelle Fotografie dar, und sie ist beeinflusst durch die aktuellen Geschehnisse. Geplant ist, eine solche Befragung in etwa 3 Jahren zu wiederholen, damit man dann auch die Entwicklung feststellen kann. Der Präsident bedankt sich abschliessend bei Lukas Golder für seine Präsentation und bei der gfs.bern für die bemerkenswerte Arbeit. Er kommt anschliessend zum dritten Teil seiner Informationen.

Visionen für die FMH/Denkanstösse für die Zukunft Auf der Basis der 4 von gfs.bern aufgestellten Arbeitsthesen wird sich der Zentralvorstand Überlegungen zur Zukunft des schweizerischen Gesundheitssystems und zu Position und Rolle der FMH machen, um der Ärztekammer nächstes Jahr ein aktualisiertes Strategiepapier vorlegen zu können. Als Vorbereitung darauf fand zum einen im Februar eine breitgefächerte Umfrage bei Fachleuten des Gesundheitswesens statt, welchen man 5 Fragen stellte. Von 37 kontaktierten Personen haben 22 geantwortet, die Ergebnisse werden im Sommer in der SÄZ publiziert. Zum andern wurden in einer Klausurtagung mit einer kleinen Arbeitsgruppe folgende Statements entwickelt:

Statement 1: «Der Beruf des Arztes/der Ärztin muss eine moderne, unverwechselbare Identität erhalten.»

Statement 2: «Das Arztbild muss seine Integrität und Glaubwürdigkeit behalten.»

Statement 3: «Die Einstellung der Patientinnen und Patienten muss mitberücksichtigt werden.»

Statement 4: «Unser Sozialstaat muss erhalten bleiben.»

Statement 5: «Der Standpunkt der Ärzteschaft muss in die Politik einfliessen.»

Statement 6: «Leadership tut Not.»

Der Präsident bittet anschliessend Michel Matter/ GE, seinen Antrag Nr. 2 zu Traktandum 5 zu erläutern. Der Antragsteller hält fest, dass der Einsatz der FMH für die beruflichen und wirtschaftlichen Interessen der Mitglieder erst im 7. Ziel des Strategiedokumentes vom letzten Dezember formuliert ist. Die FMH sei aber die Gewerkschaft der Ärztinnen und Ärzte und müsse diese Rolle vollumfänglich wahrnehmen. Deshalb beantragt er, dass die Ziele 7.1., 7.2. und 7.3. gestrichen und ein neues Ziel 1.1. mit folgendem Wortlaut eingefügt wird: «Die FMH setzt sich in erster Linie für die beruflichen und wirtschaftlichen Interessen ihrer Mitglieder ein.»

Christine Romann/ZV findet es schade, wenn die erwähnten Ziele ersetzt würden. Christoph Bosshard/ VSAO erachtet es als nicht ideal, dieses Ziel an die erste Stelle zu setzen. Der Präsident, der im Grundsatz der Meinung ist, dass man den Antrag entgegennehmen könnte, präzisiert, dass es nicht die Meinung sei, das Papier neu zu schreiben. Allerdings stellt er fest, dass 
lassen. Er sei gerne bereit, sofern gewünscht, dem Zentralvorstand seine Mitarbeit für diesen Wandlungsprozess anzubieten. Daniel Herren/ZV sieht sich als eines der neuen ZV-Mitglieder ebenfalls etwas hilflos diesen Forderungen gegenüber. Er wäre froh, wenn der Antragsteller der Unzufriedenheit, die aus seiner Präsentation zu spüren ist, mit konkreteren Forderungen und auch Ideen, wie das umgesetzt werden könnte, Gestalt geben würde.

In der Folge äussern sich einige Delegierte, dass, obwohl als konstruktive Kritik gemeint, die Präsentation von P. Wiedersheim als Angriff auf Personen oder als Misstrauensvotum gegenüber dem ZV empfunden wurde.

Urs Stoffel/ZH bittet, jetzt nicht auf den Meldeläufer zu schiessen. Er bedauert, dass der Zentralvorstand sich nie über die Schwierigkeiten bei der Umsetzung der Führungsmodelle habe verlauten lassen.

Hp. Kuhn/Stv. Generalsekretär macht darauf aufmerksam, dass die Formulierung des Antrags wie von P. Wiedersheim jetzt präsentiert, nicht ganz dem Wortlaut entspricht, wie er im Antrag Nr. 3 zu Traktandum 5 steht. $P$. Wiedersheim besätigt auf Anfrage des Präsidenten, dass der heute projizierte Wortlaut gilt; er ist weniger strikt und lässt eine gewisse Variabilität zu, gerade auch für die Rückmeldungen, die heute aus dem ZV gekommen seien. Selbstverständlich ist ihm klar, dass es eine gewisse Flexibilität braucht, um diese Anpassungen jetzt vorzunehmen und zu ändern, was man kann, aber auch, was sich als falsch erweist, zurückzunehmen. Genau da wäre die DV der geeignete Ort, denn sie hatte auf Antrag der Ärztekammer eine Arbeitsgruppe mit der Ausarbeitung dieser Führungsmodelle beauftragt.

Nachdem das Wort nicht weiter verlangt wird, schlägt der Präsident namens des Zentralvorstandes vor, den Antrag als eine Anregung und einen Aufruf entgegenzunehmen, in der Konkretisierung der Führungsmodelle voranzukommen. Der ZV wird P. Wiedersheim/VEDAG an eine Sitzung einladen, um mit ihm über das, was in seinen Augen nicht stimmt, konkreter zu diskutieren. Die Delegiertenversammlung wird vom Zentralvorstand regelmässig auf dem Laufenden gehalten.

Auf Nachfrage des Präsidenten erklärt sich P. Wiedersheim/VEDAG mit diesem Vorgehen einverstanden.
Zum Abschluss dieses Traktandums bittet der Präsident die Ärztekammer, der Form halber über den $A n$ trag des ZV Nr. 1 zu Traktandum 5 abzustimmen. Der Antrag beinhaltet die (erfolgte) Information über die Umsetzungsschritte der im Dezember 2008 genehmigten politischen und strategischen Zielsetzungen und über die Mitgliederbefragung sowie die nächste Vorlage der Jahresziele an der ordentlichen ÄK 2010.

Die Ärztekammer genehmigt den Antrag einstimmig, ohne Gegenstimmen und bei 18 Enthaltungen.

(An dieser Stelle werden die Informationen aus dem Ressort «Tarife und Verträge» eingeschoben: s. Trakt. 17.)

Um 13.08 Uhr unterbricht der Präsident die Sitzung für eine kurze Mittagspause.

Die Sitzung wird um 13.54 Uhr wieder aufgenommen.

\section{Budget 2010}

Der Präsident übergibt das Wort dem Generalsekretär zur Präsentation des Budgets 2010.

Daniel Herzog weist die Delegierten zunächst auf die verschickten Unterlagen hin, Budget 2010 (Zusammenfassung mit Vorjahresvergleich), Detailbudget FMH und Abteilungen, Bemerkungen zum Budget, Budget AWF, um dann auf die wichtigsten Punkte näher einzutreten. Das Budget weist ein kleines Defizit von CHF -272000.00 aus (Vorjahresbudget CHF -359000.00). Davon werden der AWF-Reserve CHF -138000.00 zugewiesen, so dass der FMH noch ein Defizit von CHF -134000.00 verbleibt. Es ist mit vergleichbaren Aufgaben wie im laufenden Jahr zu rechnen, weshalb die Mitgliederbeiträge gegenüber 2009 unverändert bleiben. Die Sonderbeiträge reduzieren sich für 2010 um CHF 29.00 (der Sonderbeitrag von CHF 25.00 für die HPC fällt weg, derjenige für die NAKO vermindert sich um CHF 4.00).

\section{Festsetzung des Grundbeitrages 2010 sowie allfälliger Sonderbeiträge}

Nachdem das Wort nicht verlangt wird, präsentiert der Generalsekretär die dem Budget 2010 zugrunde gelegten Mitgliedergrundbeiträge für die verschiedenen Kategorien. (s. Tab. 1)

Der Präsident bittet anschliessend die Delegierten um Abstimmung über den Antrag des Zentralvorstands Nr. 1 zu Traktandum 6.

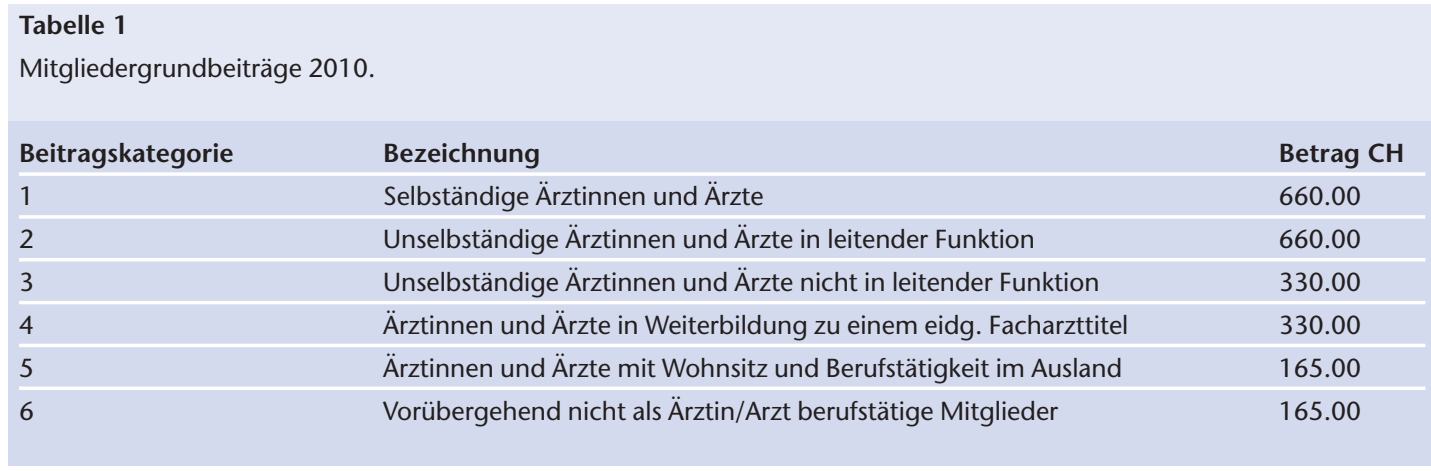


Die Ärztekammer genehmigt das Budget 2010 mit den präsentierten Mitgliedergrundbeiträgen mit 146 Stimmen, ohne Gegenstimmen und bei 1 Enthaltung.

\section{Sonderbeitrag NAKO (Newlndex)}

E. Gähler/ZV unterstreicht, von welch unschätzbarem Wert die Zahlen aus der NAKO für die FMH, die KKA und einen Grossteil der Fachgesellschaften für Vertragsverhandlungen, Monitorings (z.B. Analysenliste) und für die Validierung von Zahlen der santésuisse sind. Je nach Auftrag werden die gewünschten Zahlen durch die NAKO aufbereitet. Er ruft die Ärztekammer dazu auf, diesen wichtigen Sonderbeitrag zu genehmigen. Das Wort wird nicht weiter verlangt. Der Präsident bringt deshalb den Antrag des Zentralvorstands Nr. 2 zu Traktandum 6 zur Abstimmung.

Der NAKO-Sonderbeitrag von CHF 36.00, erhoben ausschliesslich bei den Mitgliedern der Beitragskategorien 1 und 2, wird mit 138 Stimmen, 1 Gegenstimme und 9 Enthaltungen angenommen.

(An dieser Stelle werden die Informationen aus dem Ressort Medical Education eingeschoben: s. Traktandum Nr. 18).

\section{Anträge zu Gremien und Mitgliedschaften}

\section{Bestätigungen für die \\ FMH-Delegiertenversammlung}

Der Präsident präsentiert die Liste der zur Bestätigung beantragten Delegierten und der Ersatzdelegierten für die FMH-Delegiertenversammlung (Antrag Nr. 1a zu Traktandum 7.1.). Die Liste hat gegenüber dem mit der Einladung versandten Antrag Nr. 1 zu Traktandum 7.1. einige Ergänzungen erfahren.

Für Th. Heuberger/BE ist gegen die aufgeführten ordentlichen Delegierten nichts einzuwenden, sie können in globo bestätigt werden. Als Vorsitzender der Delegiertenversammlung stellt er sich jedoch die Frage, ob gegen die von der fmCh vorgeschlagene Ersatzdelegierte Christiane Roth/SGGG, die seines Wissens im Verwaltungsrat der Helsana sitze, seitens der Ärztekammer nicht Vorbehalte bestünden. M. Trutmann/Generalsekretär der fmCh bestätigt, dass Ch. Roth von der fmCh in vollem Wissen um diese Interessenbindung einstimmig als Ersatzdelegierte nominiert worden sei und der fmCh sehr daran liege, dass Ch. Roth diese Funktion übernehme. Ch. Roth erklärt ihrerseits, dass sie wohl Verwaltungsrätin der Helsana sei, sich aber gewohnt sei, bei allenfalls entstehenden Interessenkonflikten in den Ausstand zu treten. Sie sei sich ebenfalls gewohnt und habe sich auch bisher immer daran gehalten, die Vertraulichkeit von Informationen zu wahren. Th. Heuberger/BE plädiert dafür, die Bestätigung von Ch. Roth separat durchzuführen. Der Präsident schlägt vor, die Liste der ordentlichen DV-Delegierten in globo zu bestätigen und über das Ersatzdelegiertenmandat von Ch. Roth schriftlich abzustimmen. Jürg Nadig/Med. Onkologie/SFSM macht beliebt, dass die Interessenbindungen der nominierten Delegierten/Ersatzdelegierten vor der Wahl vorliegen.
Es folgt eine hitzige, von einigen als unwürdig erachtete Diskussion, in deren Verlauf M. Reber/LU u. a. feststellt, dass die Vertreter der Krankenkassen nicht nur im Ständerat, sondern nun auch noch in der FMHDelegiertenversammlung sitzen. Diese Unterstellung kontert der Präsident mit scharfen Worten und weist darauf hin, dass Ch. Roth mit ihrer Erfahrung und ihren Charaktereigenschaften, die sie über Jahre in ihrer Tätigkeit im FMH-Generalsekretariat bewiesen habe, durchaus fähig sei, die Anliegen der Ärzteschaft in ein Gremium hineinzutragen, wo diese sonst nicht gehört werden. M. Trutmann/fmCh weist darauf hin, dass es nicht nur Interessenkonflikte mit Krankenkassen, sondern manchmal auch mit kantonalen Regierungen, mit der Industrie oder mit Spitälern gibt. Wenn diese Interessenbindung von Ch. Roth nun als problematisch erachtet werde, dann gelte dies auch für alle anderen Interessenbindungen. Die fmCh werde nun die Interessenbindungen sämtlicher DV-Delegierten genau unter die Lupe nehmen. M. Vischer/ORL findet die Diskussion merkwürdig und spricht Ch. Roth sein volles Vertrauen aus. Nachdem Ch. Roth darauf verzichtet, noch etwas beizufügen, orientiert der Präsident nochmals über das weitere Vorgehen: Während die Ärztekammer nun über die Liste der vorgeschlagenen ordentlichen DV-Delegierten beschliesst, verteilt das Sekretariat den stimm- und wahlberechtigten Delegierten den Wahlzettel für die schriftliche Abstimmung. Dies könnte etwas Zeit in Anspruch nehmen, weil ein Wahlgang an der heutigen Sitzung nicht vorgesehen war.

Die Ärztekammer bestätigt die folgenden ordentlichen DV-Delegierten mit überwältigender Mehrheit, bei einer Enthaltung:

Christian Seiler, Bern/SFSM, Peter Leuenberger, Genf/ FMCH, Marc-Alain Panchard, Vevey/KHM, Kurt Schreier, Gersau/VEDAG.

Sobald die Wahlzettel verteilt sind, erläutert der Generalsekretär das Prozedere: Wer Ch. Roth/SGGG als Ersatzdelegierte in die Delegiertenversammlung wählen möchte, schreibt auf den Wahlzettel ihren Namen. Wer Ch. Roth nicht wählen will, macht auf den Wahlzettel einen Strich. Die Stimmenzähler sammeln mit den Wahlurnen die Wahlzettel ein, diese werden im Stimmbüro ausgezählt.

Das zunächst verkündete Wahlresultat ist nicht korrekt, der Leiter des Stimmbüros war nicht mehr im Plenarsaal, als der Generalsekretär den Abstimmungsmodus verkündete. Die leeren oder mit einem Strich markierten Wahlzettel wurden deshalb irrtümlicherweise als ungültig gezählt. Die Stimmzettel werden nochmals ausgezählt.

Der Generalsekretär gibt das Wahlergebnis bekannt:

Eingegangene Stimmzettel 150, gültig 150, Quorum 76. Christiane Roth, vorgeschlagen als Ersatzdelegierte der fmCh, hat 46 Stimmen erhalten und ist damit nicht gewählt.

Die fmCh muss einen neuen Vorschlag unterbreiten. 


\section{GPK-Amtszeitverlängerung}

Roland Schwarz/Präsident GPK erläutert kurz den Antrag der GPK Nr. 1 zu Traktandum 7.2.: Philippe Rheiner, bei den Gesamterneuerungswahlen der FMH-Organe an der ordentlichen Ärztekammer 2008 als neues Mitglied der Geschäftsprüfungskommission gewählt, hat die statutarisch vorgesehene Altersgrenze für Mitglieder in Organen und Kommissionen erreicht. Sowohl der GPK als auch der SMSR, die ihn nominiert hat, war es nicht bewusst, dass ihr Kandidat bei seiner Wahl bereits 67 Jahre alt war. Laut Statuten kann das zuständige Wahlgremium die Altersgrenze jedoch in Ausnahmefällen hinausschieben. Der GPK-Präsident macht deshalb beliebt, dass Philippe Rheiner das vor einem Jahr angetretene Amt bis zum Ende der laufenden Amtsperiode im Jahr 2012 weiterführen kann. Christoph Bosshard/VSAO hält fest, dieser Antrag habe den VSAO befremdet, weil die in Statuten und Reglementen festgeschriebenen Spielregeln wieder einmal überdehnt würden. Dass so etwas einmal anlässlich einer Wahl übersehen werde, sei an sich verständlich. Der VSAO bittet aber darum, entweder Statuten oder Reglemente zu ändern oder aber deren Inhalt auch zu leben. Philippe Rheiner/GPK erinnert daran, er habe, als er zur Wahl stand, auf sein Alter hingewiesen, was aber offensichtlich untergegangen sei. Nachdem keine weiteren Wortmeldungen erfolgen, lässt der Präsident über den Antrag abstimmen.

Die Ärztekammer heisst den Antrag Nr. 1 zu Traktandum 7.2. bei einer Gegenstimme und 11 Enthaltungen gut. Philippe Rheiner kann somit sein Amt als GPKMitglied bis zu den Gesamterneuerungswahlen im Jahr 2012 weiterführen.

\section{Sitzverteilung Ärztekammer}

Der Präsident erteilt Hans Kurt/SGPP, FMPP das Wort zur Vorstellung seines Antrags Nr. 1 zu Traktandum 7.3.: Gemäss Statuten wird die Sitzverteilung für die Ärztekammer alle vier Jahre festgelegt, letztmals Anfang 2008 (Anm. der Protokollführerin: Art. 26 und 27 FMHStatuten und Art. 6 und 8 Abs. 1 GO). Im vergangenen Dezember sind zwei neue Fachgesellschaften von der Ärztekammer anerkannt worden, was dazu geführt hat, dass die SGPP einen ihrer Delegiertensitze in der ÄK verloren hat. Für den Antragsteller ist diese Situation sowohl statutarisch wie auch in Bezug auf eine faire Verteilung der Sitze unbefriedigend. Er ersucht deshalb den Zentralvorstand zu prüfen, wie man dieses Problem lösen könnte. Sein Vorschlag: Neu aufgenommene Fachgesellschaften müssen bis zum Ablauf der vierjährigen Amtsdauer warten, bis ihnen die Vertretung in der Ärztekammer zugestanden wird, oder aber die Sitzverteilung in der ÄK wird jeweils Ende Jahr entsprechend den effektiven Mitgliederzahlen der Fach- und Kantonalgesellschaften bestimmt.

Der Präsident erklärt, diesen Antrag namens des Zentralvorstandes entgegenzunehmen, zusammen mit dem Rechtsdienst die Situation anzuschauen und einen Vorschlag auszuarbeiten. Hp. Kuhn/Stv. General- sekretär und Leiter Rechtsdienst weist darauf hin, dass bei der vorgeschlagenen «Jahreslösung» neben dem Arbeitsanfall sich eine weitere Schwierigkeit ergibt: Grundidee war, dass Ärztekammerdelegierte auf 4 Jahre gewählt werden. Es wird dann dazu führen, dass Ärztekammerdelegierte, obschon gewählt, plötzlich ihren Sitz verlieren. Christoph Bosshard/VSAO macht darauf aufmerksam, dass viele Ärztinnen und Ärzte in mehreren Organisationen gleichzeitig Mitglied sind, ausser einem Grossteil der VSAO-Mitglieder. Er möchte deshalb beliebt machen, dass man sich bei der Verteilung der Sitze nur auf die Basisorganisationen und die dort beheimateten Mitglieder bezieht, weil sonst diejenigen, die in einer Basisorganisation und auch noch in verschiedenen Fachgesellschaften Mitglied sind, entsprechend übervertreten sind. Laut Jacques de Haller wird auch dieses Votum in die Überlegungen einbezogen werden.

Nachdem das Wort nicht weiter verlangt wird, bestätigt der Präsident nochmals gegenüber dem Antragsteller, dass der Zentralvorstand den Antrag entgegennimmt und einen Vorschlag ausarbeiten wird. Der Antragsteller Hans Kurt zeigt sich damit einverstanden.

\section{Fähigkeitsausweis und FMH-Beitrittspflicht}

Nach der geltenden Weiterbildungsordnung ist zur Erlangung eines Fähigkeitsausweises die FMH-Mitgliedschaft obligatorisch. Der Antrag Nr. 1 zu Traktandum 7.4. wollte dies in Frage stellen. François Dami/VSAO erklärt, dass sein Antrag noch verbesserungsfähig sei, weshalb der VSAO entschieden habe, ihn zurückzuziehen. Wer, sei es ein Verband oder ein Individuum, bei den Überlegungen gerne mitarbeiten möchte, sei willkommen und möge mit ihm Kontakt aufnehmen.

\section{Liste der Ärztekammerdelegierten}

Der Antragsteller Jean-Pierre Pavillon/VD zeigt sich befremdet darüber, dass ihm vor einigen Monaten auf entsprechende Anfrage hin das Generalsekretariat mit dem Hinweis auf Vertraulichkeit die Liste mit den Namen der aktuellen Ärztekammerdelegierten verweigert habe. Er kenne kein demokratisches Land, in welchem die Zusammensetzung der Legislative als Staatsgeheimnis betrachtet werde. Er verlangt deshalb mit seinem Antrag Nr. 1 zu Traktandum 7.5., dass den in der ÄK vertretenen Organisationen auf Anfrage die aktualisierte Liste mit den vollständigen Adressangaben (Postadresse, Telefonnummer, Faxnummer und E-Mail-Adresse) zur Verfügung gestellt werde. R. Oppliger/SGAI unterstützt im Sinne von mehr Demokratie den Antrag. Dies habe die FMH bitter nötig, was insbesondere das Vernehmlassungsprozedere bezüglich der Komplementärmedizin deutlich gezeigt habe. Es sei inakzeptabel, einen demokratischen Prozess auf dem Altar der politischen Opportunität zu opfern. Die SGAI hoffe, dass die Geschäftsprüfungskommission Licht in die Verantwortlichkeiten bringen werde, denn hier stehe die Glaubwürdigkeit der ganzen FMH auf dem Spiel. Ch. Bosshard erklärt namens des VSAO, den 
Antrag in dem Sinne zu unterstützen, als die E-MailAdressen den in der ÄK vertretenen Organisationen zugänglich gemacht werden. Der VSAO lehnt aber kategorisch ab, dass die vollständige Adresse mit Telefon, Fax etc. praktisch öffentlich zugänglich ist. H.-U. Würsten/VLSS unterstützt seinen Vorredner, was die E-Mail-Adressen betrifft. Irgendwo beginne jedoch die Privatsphäre. Jean-Pierre Pavillon kann sich dem anschliessen und bestätigt, dass nur die Namen und die berufliche E-Mail-Adresse bekannt gegeben werden sollen. Hp. Kuhn präzisiert zuhanden des Protokolls, dass der Antrag geändert wurde, indem jetzt nur noch die Rede von der E-Mailadresse ist (also keine Postadresse, Telefonnummer, Faxnummer mehr!). J.-P. Pavillon bestätigt dies. Marcel Stampfli/BE äussert ebenfalls Bedenken, was die Herausgabe der Adressen betrifft. Andererseits scheint ihm wichtig, dass die Fachgesellschaften mit allen Ärztekammerdelegierten kommunizieren können. Er stellt deshalb den Antrag, dass die in der ÄK vertretenen Organisationen via FMHGeneralsekretariat Mitteilungen per Mail an die ÄKDelegierten verschicken lassen können. Ch.-A. FavrodCoune macht auf den in der Schweiz geltenden Datenschutz aufmerksam, wonach Empfänger von solchen E-Mail-Mitteilungen kein Recht haben, die elektronischen Adressen für andere Zwecke zu verwenden. Der Präsident präzisiert, dass durch Einfügen der Empfänger-E-Mail-Adressen ins «BCC» einer missbräuchlichen Weiterverwendung schon gewisse Grenzen gesetzt werden kann. Nachdem das Wort nicht weiter verlangt wird, bringt der Präsident die beiden Anträge zur Abstimmung.

Zunächst genehmigt die Ärztekammer den Antrag von Marcel Stampfli/BE mit grosser Mehrheit.

Anschliessend wird der auf die Herausgabe von Namen, Vornamen und E-Mail-Adresse abgeänderte Antrag Nr. 1 zu Traktandum 7.5. mit grosser Mehrheit, bei 9 Gegenstimmen und 12 Enthaltungen, angenommen.

\section{Einsitznahme des VSAO in den Zentralvorstand der FMH}

Ch. Bosshard/VSAO erläutert seinen zu Beginn der Sitzung eingereichten Antrag. Anlässlich der letzten Gesamterneuerungswahlen war es dem VSAO nicht möglich, einen Kadidaten oder eine Kandidatin für den Zentralvorstand zu stellen. Jetzt aber verfügt der VSAO über jemanden, der sich dafür interessiert. Der VSAO plädiert deshalb dafür, dass ihr Kandidat ab sofort und bis zu den Gesamterneuerungswahlen 2012 Gastrecht im ZV erhält. Die Geschwindigkeit der politischen Ereignisse mache kürzere Wege zwischen den operativen Gremien des VSAO und der FMH notwendig. Der Präsident hält dafür, dass es für die FMH wichtig ist, wenn ihr oberstes Führungsorgan so repräsentativ wie möglich ist, und er stellt fest, dass der Zentralvorstand zu seinen Sitzungen Gäste einladen kann. Ch. Bosshard/ VSAO gibt auf entsprechende Nachfrage bekannt, dass es sich bei dem Kandidaten um Gerd Prinzen, Präsident der VSAO-Sektion Innerschweiz, handle. Brigitte
Muff/ZH erkundigt sich, ob der VSAO-Vertreter auch auf eine Entschädigung verzichte. Der Präsident, der diese Frage auch gestellt hat, bekräftigt, dass der VSAO dies übernehme, bis der Vertreter des VSAO ordnungsgemäss gewählt werde.

Nachdem keine weiteren Wortmeldungen erfolgen, bittet der Präsident die Ärztekammer, ihre Meinung zum Vorschlag des VSAO abzugeben. Die Meinung der Ärztekammer sei für den ZV wichtig.

Für den Vorschlag des VSAO spricht sich die Ärztekammer mit grosser Mehrheit, bei 12 Gegenstimmen und 15 Enthaltungen, aus.

Der Präsident hält fest, dass der Zentralvorstand mit dem VSAO besprechen werde, wie der Vorschlag am besten umzusetzen sei.

\section{Revision Statuten \\ (Fristen für DV-Rundschreiben)}

Hp. Kuhn/Stv. Generalsekretär erklärt, dass der Wunsch aus der Delegiertenversammlung gekommen sei, die Fristen für die Einreichung von Traktanden und den Versand der Einladung zu verkürzen. Aus praktischen Gründen soll die Einladung neu wenigstens 1 Woche vor der DV-Sitzung versandt werden (Art. 38 Abs. 2), und der DV-Vorsitzende, die Mitglieder des $Z V$ sowie die DVDelegierten können [neu] bis spätestens 14 Tage vor der Sitzung schriftlich Anträge auf Behandlung eines in die Kompetenz der DV fallenden Geschäftes stellen (Art. 38a Antragsrecht).

Ch.-A. Favrod-Coune möchte eine Präzisierung zu Art. 38a. Im französischen Text lautet die Formulierung: «... peuvent présenter des propositions demandant que l'AD traite une affaire de sa compétence.» Diese Formulierung könnte vermuten lassen, dass die DV einen Antrag zurückweisen kann. Er möchte zuhanden des Protokolls festhalten, dass die DV verpflichtet sei, einen solchen Antrag auf Behandlung aufzunehmen. Hp. Kuhn versichert seinen Vorredner, dass dies genau so gemeint sei und die DV dies bisher auch immer so gehandhabt habe. Er bittet, dies im Protokoll auch so festzuhalten.

Der Antrag Nr. 1 zu Traktandum 8 wird einstimmig mit 138 Stimmen, ohne Gegenstimmen und ohne Enthaltungen, angenommen.

\section{Revision Geschäftsordnung (Einführung Postulat)}

Th. Heuberger/BE erläutert seinen Antrag Nr. 1 zu Traktandum 9: Es kommt immer wieder vor, dass die Ärztekammer eine Idee formuliert und die Richtung aufzeigt, in welche diese Idee gehen könnte, also ein klassisches Postulat. Heute bleibt nur die Alternative, einem Antrag zuzustimmen oder ihn abzulehnen. Der Vorschlag, welcher der Ärztekammer vorliegt, sieht eine Ergänzung in Art. 11 und 19 der Geschäftsordnung (GO) wie folgt vor: Art. 11 Gang der Verhandlungen der ÄK [Art. 35 FMH-Statuten], [neu] « $5^{\text {bis: }}$ Jeder Delegierte, jedes ZV-Mitglied und der Generalsekretär haben zudem das Recht, ein Postulat einzureichen. Dieses beauf- 
tragt den $Z V$, zu prüfen und Bericht zu erstatten, ob ein Entwurf zu einer vereinsrechtlichen Regelung in der FMH vorzulegen oder eine Massnahme zu treffen sei.»

Art. 19 Verhandlungen [DV], [neu] « $2^{\text {bis }}$ : Jeder Delegierte, jedes ZV-Mitglied und der Generalsekretär haben das Recht, Anträge zu den zur Diskussion stehenden Traktanden zu stellen. Sie können zudem ein Postulat einreichen (Art. 11 Abs. $5^{\text {bis }} \mathrm{GO}$ )».

Nachdem das Wort nicht weiter verlangt wird, bringt der Präsident den Antrag zur Abstimmung.

Die Ärztekammer genehmigt Antrag Nr. 1 zu Traktandum 9 einstimmig mit 141 Stimmen, ohne Gegenstimmen und Enthaltungen.

\section{Übernahme von Richtlinien der SAMW in die FMH-Standesordnung}

Der Ärztekammer wird beantragt, die folgenden Richtlinien der Schweizerischen Akademie für Medizinische Wissenschaften (SAMW) in die Standesordnung der FMH zu übernehmen: «Reanimationsentscheidungen», «Verwendung von Leichen und Leichenteilen in der medizinischen Forschung», «Lebendspende von Organen», «Medizinische Behandlung und Betreuung von Menschen mit Behinderung». Der Antrag Nr. $1 \mathrm{zu}$ Traktandum 10 wurde seit Versand der Ärztekammereinladung mit der Richtlinie «Erstellen von Patientenverfügungen und Umsetzung im medizinischen Alltag» ergänzt (neu Antrag Nr. 1 a zu Traktandum 10). Der Text wurde den Delegierten im Nachversand zugestellt.

Ein weiterer Antrag, Nr. 2 zu Traktandum 10, wurde von Pierre-Alain Schneider/GE eingereicht. Der Präsident bittet ihn um Erläuterung. Nach Meinung des Antragstellers muss die Standesordnung ein einfacher und praktischer, allen Ärztinnen und Ärzten bekannter Text bleiben. Mit der Zunahme der in Art. 18 erwähnten Anhangrichtlinien wird die Standesordnung aber immer schwerfälliger und komplexer. Die Grundprinzipien sollen deshalb in die Artikel übernommen werden und den Anhangrichtlinien eine erläuternde Rolle zugewiesen werden. Er verzichtet auf eine Abstimmung, bittet aber den Zentralvorstand, seinen Vorschlag zu prüfen. Der Präsident nimmt diesen Vorschlag zuhanden des Zentralvorstandes entgegen.
Lukas Wagner/BL beantragt, über die Aufnahme der Richtlinie bezüglich der Patientenverfügungen eine separate Abstimmung durchzuführen.

Der Präsident folgt diesem Antrag und ersucht die Ärztekammer, über die ersten 4 SAMW-Richtlinien im Antrag Nr. 1a des ZV zu Traktandum 10 («Reanimationsentscheidungen», «Verwendung von Leichenteilen in der medizinischen Forschung», «Lebendspende von soliden Organen» und «Medizinische Behandlung und Betreuung von Menschen mit Behinderung») abzustimmen.

Die Ärztekammer stimmt der Aufnahme dieser vier SAMW-Richtlinien in die FMH-Standesordnung mit grosser Mehrheit, bei 1 Gegenstimme und 12 Enthaltungen, $z u$.

Der Präsident erteilt anschliessend $L$. Wagner/BL das Wort. Dieser plädiert dafür, die SAMW-Richtlinie betreffend Patientenverfügungen heute noch nicht in die Standesordnung aufzunehmen, sondern vorher eine gezielte Meinungsbildung durchzuführen und die kantonalen Ethikkommissionen zu befragen. Christian Kind/SGP erinnert daran, dass diese Richtlinien von Januar bis Ende März in Vernehmlassung waren und dass dazu über 80, teilweise sehr ausführliche Stellungnahmen, auch von Ethikkommissionen, eingegangen seien. Nach Hp. Kuhn/Stv. Generalsekretär und Leiter Rechtsdienst macht es Sinn, sich das Verschieben oder Nichtverschieben in Ruhe zu überlegen. Der definitive Text der Richtlinie liegt erst seit ein paar Tagen vor, es wurden auch Anliegen der FMH, die in Richtung der Anliegen von L. Wagner gehen, aufgenommen. Die Aufnahme jetzt birgt einen wichtigen politischen Vorteil in sich: Im Erwachsenenschutzgesetz ist festgehalten, dass Patientenverfügungen grundsätzlich gelten sollen. Dies ist für ihn der wichtige Punkt: Das Papier der SAMW ist ein gutes Argument dafür, dass nicht jede irgendwie entstandene und formulierte Patientenverfügung rechtlich verbindlich sein soll. In diesem Sinne ist es eine gesunde, kritische Reaktion gegenüber dem Gesetz, für ihn wäre es deshalb politisch wünschbar, wenn sich die FMH jetzt schon dahinterstellt, als gesundes Gegengewicht zu den etwas naiven Vorstellungen des Gesetzgebers. Angesichts der Inflation an Ver-

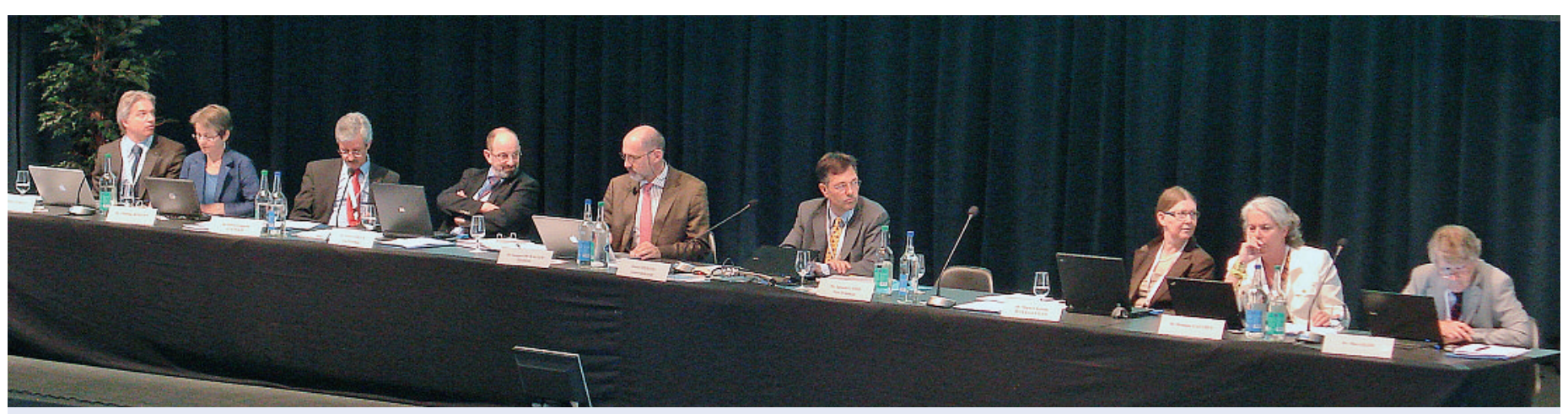

Der Zentralvorstand der FMH steht Red und Antwort, v.I.n.r.: Daniel Herren, Christine Romann, Pierre-François Cuénoud, Ernst Gähler, Jacques de Haller, Daniel Herzog (Generalsekretär), Marie-Christine Peter-Gattlen, Monique Gauthey, Max Giger. Abwesend: Ignazio Cassis. 
fügungen empfiehlt Hans Ulrich Iselin/AG, die FMH solle sich darüber Gedanken machen, welche Formen von Verfügungen sinnvoll seien. Laut Hp. Kuhn ist dies gerade das implizite Ziel der hier vorliegenden Richtlinie. Müsste die FMH ein Rating machen, würde dies einerseits sehr viel Geld kosten, andererseits wäre das auch politisch nicht sehr sinnvoll.

Nach weiteren Diskussionen bittet der Präsident die Ärztekammer, über den Antrag von L. Wagner/BL auf Verschiebung der Aufnahme der SAMW-Richtlinie bezüglich Patientenverfügungen abzustimmen.

Eine klare Mehrheit der Ärztekammer spricht sich für Annahme des Antrags aus.

\section{Änderung des Reglements der Geschäfts- prüfungskommission/Genehmigung eines GPK-Entschädigungsreglementes}

Der Präsident übergibt das Wort an Roland Schwarz/ Präsident der GPK zur Erläuterung seines Antrags. Die GPK beantragt aufgrund der in der praktischen Tätigkeit gemachten Erfahrungen, das Reglement der GPK in folgenden Artikeln anzupassen: Art. 1.3. Abs. 1/ Stellung innerhalb der FMH: Der Wortlaut soll ergänzt werden mit der Formulierung, die GPK sei ausschliesslich der Ärztekammer rechenschaftspflichtig und ihr gegenüber verantwortlich. Art. 4.7./Beizug Dritter soll wie folgt ergänzt werden: «Die GPK entscheidet, ob sie auch für administrative Belange Dritte beizieht oder die Dienste des Generalsekretariates in Anspruch nimmt.» Art. 5.7. Abs. 2/Vorgehen bei ausserordentlichen Fällen soll ergänzt werden mit einem Abs. 2: «Müssen Dritte zur Eruierung des Sachverhaltes beigezogen werden und ist absehbar, dass das Budget der GPK überschritten wird, entscheidet die GPK, ob dieser Mehraufwand angesichts der Bedeutung der Sache und der möglichen Folgen für die FMH zwingend ist. Der Beschluss der GPK ist durch die Ärztekammer im Rahmen der Genehmigung der Jahresrechnung als Nachkredit zu genehmigen.» Art. 5.8./Unterstützung durch das Generalsekretariat soll mit dem Beizug Dritter ergänzt werden. Art. 8/Entschädigung/finanzielle Ressourcen soll einen neuen Abs. 1 erhalten: «Auf Antrag der GPK erlässt die ÄK ein Entschädigungsreglement der GPK», und Abs. 2 soll wie folgt ergänzt werden: «... Über den Budgetantrag der GPK befindet die Ärztekammer in einem separaten Beschluss. Gleiches gilt für die Genehmigung des effektiven Aufwandes der GPK im Rahmen der Genehmigung der Jahresrechnung der FMH. Vorbehalten bleibt Ziff. 5.7. Abs. 2».

Weiter beantragt die GPK der Ärztekammer die Genehmigung des Entschädigungsreglements. Das bestehende Reglement vom 24.4.2008 soll bei den Entschädigungsansätzen geändert werden, indem Sitzungen neu nicht mehr mit einer Pauschale, sondern nach Aufwand mit einem Stundenansatz von CHF 170.00 entschädigt und die Reisezeit von und zu Sitzungen neu hälftig zum gleichen Stundenansatz angerechnet werden sollen.

Vincenzo Liguori/TI findet die vorgesehene Entschädigung für Sitzungen nach Aufwand und in ihrer Höhe in Ordnung, dass aber auch noch die Reisezeit hälftig abgegolten werden soll, findet er inakzeptabel. Damit würde ein Präzedenzfall für andere Kommissionen geschaffen.

Nachdem das Wort nicht weiter verlangt wird, bringt der Präsident den Antrag Nr. 1 zu Traktandum 11 zur Abstimmung.

Die Ärztekammer genehmigt die vorgeschlagenen Änderungen im Reglement der GPK mit grosser Mehrheit, bei 5 Gegenstimmen und 11 Enthaltungen.

Anschliessend stimmt die Ärztekammer dem geänderten Entschädigungsreglement der GPK mit 66 Stimmen, 42 Gegenstimmen und 19 Enthaltungen $z u$.

\section{HIN: Reduktion der Aktienbeteiligung}

Der Generalsekretär erläutert, weshalb der Zentralvorstand der Ärztekammer beantragt, 405 Aktien der HIN AG an Interessenten aus dem Kreis der kantonalen Ärztegesellschaften bzw. Fachgesellschaften, gegebenenfalls teilweise an die bisherigen Minderheitsaktionäre (Ärztekasse, OFAC, Zur Rose AG, BlueCare AG), zu verkaufen. Die Health Info Net AG/HIN wurde 1996 auf Initiative der FMH gegründet, die aktuell eine Aktienmehrheit von 50,5 \% hält. Im Zusammenhang mit der Vergabe des HPC-Projektes an die Swisscom kam es zu Meinungsverschiedenheiten (gegenteilige Auffassung bezüglich einer möglichen Verletzung des Aktionärbindungsvertrags durch die FMH) zwischen FMH und HIN, die auch nach mehreren Treffen nicht ausgeräumt werden konnten. Die FMH erwog deshalb, durch den Verkauf von Aktien ihre Mehrheitsbeteiligung an HIN aufzugeben, dies aus der Erkenntnis heraus, so die Konfliktsituation für beide Seiten am besten zu beseitigen. Bedingung dafür ist, dass die zum Verkauf vorgeschlagenen Aktien im Besitze der Ärzteschaft verbleiben, welche Hauptkunden und -nutzer von HIN sind. Im ersten Quartal 2009 wurden die kantonalen Ärztegesellschaften und Fachgesellschaften angefragt, ob ein Interesse an der Übernahme von HIN-Aktien zum Nominalwert von CHF 1000.00 bestehe. Seither haben sich Interessenten für rund 200 Aktien gemeldet. Es liegen noch nicht von allen befragten Gesellschaften Antworten vor, so dass davon ausgegangen werden kann, dass der grösste Teil der 405 Aktien in der Ärzteschaft platziert werden kann. Für die restlichen Aktien liegt ein Übernahmeangebot seitens der Minderheitsaktionäre vor. Da die Aufgabe der Mehrheitsbeteilung durch die FMH einem Strategiewechsel gleichkommt, unterbreitet der Zentralvorstand dieses Geschäft der Ärztekammer.

In der anschliessenden Diskussion beantragt Jürg Schlup/BE Nichteintreten auf das Geschäft. Er wird sehr nachdrücklich von Thomas Heuberger/BE unterstützt, der gar davor warnt, auf das Geschäft einzutreten. Als Begründungen, weshalb die FMH ihre Mehrheitsbeteiligung an HIN nicht aufgeben darf, werden aufgeführt, dass aktuell gut 11000 Ärztinnen und Ärzte mit HIN arbeiten und auf HIN angewiesen sind, dass eine Vielzahl von Spitälern wegen dieser grossen Anzahl ärztlicher HIN-Benutzer ebenfalls HIN einsetzen und dass es durch den Verkauf an verschiedene neue Aktionäre 
zu einer Atomisierung kommt, welche die Gefahr von wechselnden Mehrheitsverhältnissen und die Verfolgung reiner Eigeninteressen in sich birgt. Andere Votanten, wie Urs Stoffel/ZH, sind grundsätzlich auch der Meinung, dass die FMH die Aktienmehrheit behalten soll, stellen aber fest, dass «das Geschirr jetzt zerschlagen ist» und es deshalb gilt, für die mehr als 11000 HIN-Abonnenten die beste Lösung zu finden. $U$. Stoffel/ZH kündigt an, dass der VEDAG hierzu noch einen Antrag einreichen werde. Auch Ernst Gähler/ZV ist der Meinung, dass die Situation «verkachelt» ist und die Gefahr eines juristischen Hickhacks besteht (gegensätzliche juristische Gutachten der beiden Parteien zur Frage der Verletzung des Aktionärbindungsvertrages liegen vor). Wichtig sei, dass die HIN AG weitermachen könne, und er bittet deshalb, auf das Geschäft einzutreten.

Nachdem sich die Diskussion länger hinzieht, stellt Brigitte Muff/ZH den Ordnungsantrag, es sei jetzt abzustimmen. Der Ordnungsantrag wird mit grosser Mehrheit angenommen.

In der anschliessenden Abstimmung wird der Antrag von J. Schlup/BE auf Nichteintreten mit 66 JaStimmen gegen 47 Nein-Stimmen angenommen. Damit verbleiben die Aktien bei der FMH, welche die Situation mit den Minderheitsaktionären von HIN besprechen wird.

Der von U. Stoffel/ZH angekündigte VEDAG-Antrag Nr. 2 zu Traktandum 11 ist mit dem beschlossenen Nichteintreten gegenstandslos geworden.

\section{Beschlussanträge und Informationen aus den Ressorts}

\section{KVG-Revision -}

\section{gegenseitiges Beteiligungsverbot}

Markus Reber/LU hatte bereits an der a.o. Ärztekammer im Dezember 2008 für ein gegenseitiges Beteiligungsverbot plädiert. Da die vorgeschlagene Formulierung damals als zu wenig klar erachtet wurde, schlug der Präsident vor, bis zur heutigen Ärztekammer einen klaren Vorschlag seitens des Zentralvorstandes zu unterbreiten, was der Antragsteller akzeptierte.

Der Vorschlag des ZV in Antrag Nr. 1 zu Traktandum 13 lautet: «Der ZV schlägt vor, das Anliegen des gegenseitigen Beteiligungsverbots auch bei nächster Gelegenheit wieder im Parlament vorzubringen, konkret anlässlich der erwarteten Anhörung der FMH in der vorberatenden Kommission des Nationalrats (voraussichtlich Juni 2009).»

Vorgesehen wären folgende Ergänzungen im KVG: Art. 12 Abs. 5 [neu]: «Krankenkassen führen keine Einrichtung zur medizinischen Behandlung von Versicherten und beteiligen sich nicht finanziell an solchen Einrichtungen» und Art. 35 Abs. 4 [neu]: "Leistungserbringer führen keine Krankenkasse gemäss Art. $12 f$ und beteiligen sich nicht finanziell an Krankenkassen».

Der Präsident macht dem Antragsteller beliebt, den Antrag, wie er vorgeschlagen ist, anzunehmen und als einzuschlagende politische Richtung zu betrachten. Der Antragsteller erklärt sich damit einverstanden.
(An dieser Stelle werden die Informationen aus dem Ressort eHealth eingefügt: s. Traktandum 15)

\section{Daten, Demographie und Qualität}

Der Ressortleiter Daniel Herren informiert über die laufenden Arbeiten anhand der für 2009 gesteckten Ziele. Bereich Qualität:

- Führungsrolle in Qualitätstransparenz für Leistungserbringer und in Zusammenarbeit mit Koproduzenten fördern

Dies wird u.a. im Pilotprojekt Qualitätsmonitoring realisiert. Ziel ist eine (bessere) Transparenz bezüglich der Qualitätsaktivitäten der Leistungserbringer. In einer Pilotstudie, die bis 2010 läuft, werden mit drei Fachgesellschaften, der Hausarztmedizin, der Psychiatrie und der Orthopädie, Qualitätsaktivitäten erfasst und gewertet. Es folgt eine Rückmeldung an die Fachgesellschaften darüber, wie sich die Situation der Qualitätsaktivitäten in ihrer Gesellschaft darstellt. Eine Publikation in geeigneter Form ist vorgesehen, und mit den Partnern soll versucht werden, im Gesundheitswesen Anreize zu schaffen und Unterstützung zur Verbesserung anzubieten. Das hat den Zweck, eigene, selbst erhobene Daten zu gewinnen, denn nur so können datenbasierte Behauptungen entweder entkräftet oder unterstützt werden. Gegenüber der Politik können wir unser Interesse an der Darstellung der Qualität und an deren gezielten und verbesserten Transparenz glaubhaft machen. Ein erster Schritt zur Vertragserfüllung gemäss TARMED-Vertrag ist damit getan. An einem ersten Treffen im August 2009 soll den Koproduzenten im Projekt (Versicherungen, BAG, sas) der Projektstand dargestellt werden, um damit Verständnis zu schaffen und den Dialog zu fördern. Die Daten sollen ab Anfang 2010 über $m y \mathrm{FMH}$ erfasst werden können und werden anschliessend evaluiert und ausgewertet. Ursprünglich war vorgesehen, das Qualitätsmonitoring zusammen mit santésuisse durchzuführen. Die Vertragsverhandlungen scheiterten aber aus diversen Gründen, weshalb beschlossen wurde, das Projekt als reines FMH-Projekt weiterzuführen. Santésuisse hat (vielleicht deshalb) inzwischen wieder das Interesse angemeldet, als Kooperationspartner einzusteigen. Da ihre interne Organisation aber relativ kompliziert ist, soll zunächst ein Grundvertrag vereinbart werden. Dieser ist zurzeit bei santésuisse in Vernehmlassung. Es besteht berechtigte Hoffnung, dass die Zusammenarbeit zustande kommt.

Weitere, zum Teil abgeschlossene Projekte, aus welchen verschiedene Teilprojekte entstehen, sind mit der Stiftung für Patientensicherheit am Laufen. Die Stiftung geniesst eine breite politische Akzeptanz, was für eine Einflussnahme durch die DDQ von Wichtigkeit ist.

In der Begleitforschung von SwissDRG kann die DDQ in den Bereichen «Auswirkungen der neuen Tarife auf die Patientenversorgung» und «Lenkung von 
Patientenströmen» eine entscheidende Rolle übernehmen. Ein entsprechendes Konzept ist in Erarbeitung. In der Nationalen Qualitätsstrategie in der Gesundheitsversorgung will die DDQ aktiv mitwirken. Nach heutigem Stand des Wissens ist der Bericht des BAG aber immer noch nicht auf dem Tisch des EDI-Vorstehers.

Die DDQ arbeitet in verschiedenen Arbeitsgruppen mit, u. a. in der SAMW, im neu gegründeten Nationalen Verein für Qualität/NAV und in der Arbeitsgruppe Qualität von $\mathrm{H}+$.

- Netzwerk Qualität innerhalb der Ärzteschaft In den regelmässig stattfindenden Treffen mit den Qualitätsverantwortlichen der Fachgesellschaften und Dachorganisationen wird versucht, in Form von Wissensvermittlung den gegenseitigen Austausch und die Vernetzung zu fördern. Erarbeitete Grundlagenpapiere sind wichtige Arbeitsinstrumente. Auf sie kann man immer wieder zurückgreifen, wenn man zu einem bestimmten Qualitätsthema befragt wird.

- Patientensicht kennen

Hier wurde mit der Fachhochschule Bern ein wichtiges Projekt angestossen, welches bei den Qualitätsverantwortlichen der Fachgesellschaften hohes Interesse fand. Der erste Teil, eine qualitative Umfrage, ist abgeschlossen. In der SÄZ wurde darüber berichtet. Der zweite Teil, in Form einer repräsentativen Umfrage, wird in Kürze gestartet.

- Nützliche Projekte unterstützen

ReMed: das Pilotprojekt läuft weiter, ReMed wächst langsam, kontinuierlich und organisch.

Umgang mit betriebsinternen Zwischenfällen (Stiftung für Patientensicherheit)

Riskmanagement in Schweizer Spitälern (mit ETH Zürich und der Hochschule Luzern); im Juni findet die Abschlusstagung statt.

Bereich Daten/Demographie:

Hier werden Grundlagen erarbeitet, u.a.

- zu Fragen der Ärztedemographie: Bei der Bedarfsplanung und der Versorgungsqualität ist eine gute Datenlage extrem wichtig. Die Grundlage dafür wurde mit der Erweiterung der Ärztestatistik geschaffen. Geplant ist auch, die Studie «Einkommensverhältnisse der freien Ärzteschaft» allenfalls zu ergänzen und zu erweitern.

- zur ambulanten und stationären ärztlichen Leistungserbringung. Verschiedene Studien zusammen mit dem OBSAN sind am Laufen: «Medhosp» (Monitorage de médecins actifs dans les milieux hospitaliers en Suisse), «Entwicklung von Einzelindikatoren für die stationär tätigen Ärzte» Sowie «Monimed: Monitoring der in der Schweiz berufstätigen Ärztinnen und Ärzte».

Die DDQ ist eine zentrale Anlaufstelle für Auskünfte zur ärztlichen Demographie. Auf Anfrage werden Daten aufbereitet und zur Verfügung gestellt. Die Ärztestatis-

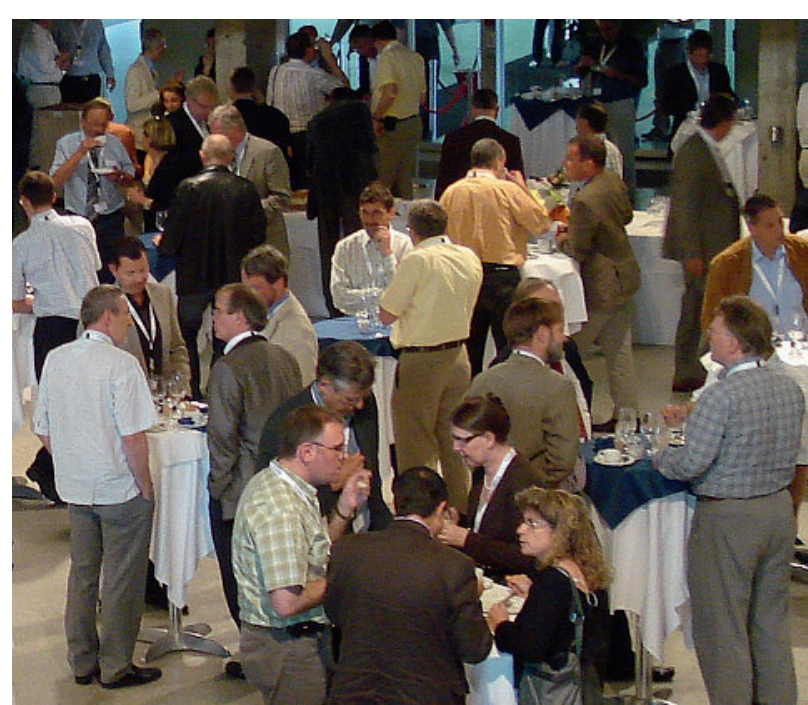

Kulinarisch gestärkt in die zweite Ärztekammer-Hälfte.

tik, die bisher nur Angaben zur Soziodemographie und zur Qualität enthielt, wurde mit Angaben zur Tätigkeit erweitert. Das Layout wurde verbessert, es gibt neu weniger Tabellen, dafür mehr Text. Die Ärztestatistik findet man auf der FMH-Website unter dem Bereich DDQ. D. Herren verweist auf den Flyer, der auf jedem Delegiertenplatz aufliegt. Er soll, neben Publikationen in der SÄZ und in den verschiedenen Verbandsorganen der Fachgesellschaften und kantonalen Ärztegesellschaften, dazu dienen, die Ärzteschaft dazu zu motivieren, die Datenqualität hochzuhalten. Der Rücklauf über $m y$ FMH betrug 15-18\% und muss unbedingt verbessert werden.

Zum Abschluss informiert D. Herren über die Umsetzung von Art. 22a KVG (Verpflichtung zur Datenlieferung bezüglich Tätigkeiten, Umsätzen in Praxen, zu Qualitätsindikatoren), ein Projekt, das auf Initiative der KKA gestartet wurde.

Obwohl der sog. Aufsichtsartikel seit 1.1.2009 in Kraft ist, hat niemand eine Ahnung bezüglich Umsetzung und Detaillierungsgrad der zu liefernden Daten. Proaktiv wurde deshalb der Kontakt zum Bundesamt für Statistik (BfS) gesucht, weil das BAG die Daten beim BfS anfordern wird. Erste Gespräche haben stattgefunden und waren sehr positiv. Das BfS ist froh und dankbar, wenn wir mithelfen, gemeinsam zu evaluieren, welche Daten von der Ärzteschaft in Richtung BAG weitergegeben werden sollen.

Christoph Ramstein/VEDAG erwähnt die vielen Kontakte und die Zusammenarbeit in Projekten, die der VEDAG in den letzten 6 Jahren mit der Abteilung DDQ hatte, und bedankt sich bei Daniel Herren sowie den Mitarbeiterinnen der Abteilung DDQ für die sowohl fachlich als auch menschlich hervorragende Arbeit. Zum Stichwort Begleitforschung SwissDRG: das Ganze läuft seiner Ansicht nach im Moment dreigleisig: Da ist einmal die parlamentarische Initiative zur Begleit- und Versorgungsforschung von Bea Heim vom 
Oktober 2008, dann der Vorstoss der KKA bei Carlo Conti, und nun hat auch die DDQ diesbezüglich etwas am Laufen. Er regt an, dass hier unbedingt Synergien geschaffen werden. Urs Stoffel/KKA kann das Votum seines Vorredners nur unterstützen. Was den KKA-Vorstoss bei Carlo Conti betrifft, weiss man eigentlich recht wenig, was damit zurzeit geschieht. Andererseits decken sich tatsächlich die Anliegen von Bea Heim mit den Forderungen der KKA bezüglich Begleitforschung und Qualitätssicherung für die Einführung der DRG. Eine gewisse Zusammenarbeit wäre deshalb sicher von Vorteil.

\section{eHealth}

Der Präsident erteilt das Wort an Monique Gauthey/ZV für die Informationen aus ihrem Ressort. M. Gauthey, die nach ihrer Wahl in den ZV vor einem Jahr das Ressort übernommen hat, zieht eine kurze Bilanz über die Arbeiten in diesem sehr komplexen Ressort.

- eHealth-Strategie der FMH

Die vom Ressort eingesetzte Arbeitsgruppe eHealth hat, basierend auf den strategischen Zielen der FMH für die Legislaturperiode 2008-2012, einen Vorschlag erarbeitet, der im Zentralvorstand diskutiert wurde. Wenige Änderungen sind durch die Arbeitsgruppe noch vorzunehmen, das Papier soll bis zum Sommer fertig gestellt und verabschiedet werden. Im Dokument werden Aussagen enthalten sein zur Mitgestaltung durch die Ärzteschaft, zur Forderung nach nutzbringenden Anwendungen und sinnvoller Unterstützung, insbesondere im Behandlungsprozess, zu einer angemessenen Integration in die ärztliche Berufsbildung sowie zum Datenschutz, zum Einfluss auf die Arzt-Patientenbeziehung und zur Finanzierung. Auf der Grundlage der eHealth-Strategie der FMH sollen Positionspapiere zu aktuellen Themen, wie u. a. Patientenidentifikation, elektronischem Patientendossier, Versichertenkarte, erarbeitet werden.

Das Koordinationsorgan eHealth ist eine gemeinsame Struktur von Bund und Kantonen, welche in einem Rahmenvertrag geregelt ist. Ziel ist die Umsetzung der vom Bundesrat verabschiedeten «nationalen Strategie eHealth» (auch «Strategie eHealth Schweiz» genannt). Die FMH ist in den verschiedenen Projektgruppen vertreten. Die dort vertretenen Positionen werden mit der Arbeitsgruppe eHealth der FMH abgestimmt. Das Organigramm des Koordinationsorgans enthält einen Steuerungsausschuss unter Leitung von Bundesrat Couchepin. Es ist verantwortlich für die Erfüllung der Strategieziele. Ihm zur Seite steht eine beratende Begleitgruppe mit den wichtigsten Interessenvertretern. Die Geschäftsstelle leitet das operative Tagesgeschäft; das Projektleitungsgremium, zuständig für die Koordination der Teilprojekte und die Erarbeitung von Entscheidgrundlagen, besteht aus den Co-Leitungen aller Teilprojekte. Die fachlichinhaltliche Arbeit erfolgt in folgenden Teilprojek- ten: «Rechtliche Grundlagen», «Standards, Architektur», «Modellversuche, PPP», «Bildung», «Online-Dienste», «Befähigung» sowie «Finanzierung, Anreizsysteme». In den Grundsätzen und Richtlinien, die als Basis für die Architektur gelten, ist z.B. zur Identifikation der Behandelnden auch die Health Professional Card/HPC enthalten. Das Ganze ist nicht als nationales Grossprojekt, sondern als Vernetzung föderaler Systeme zu verstehen. Im Vordergrund steht das Schaffen der «Voraussetzungen für die Austauschbarkeit von patientenbezogenen Dokumenten». Die drei wesentlichen Bereiche des Dokumenten- und Datenaustausches sind die «Klinischen Prozesse zwischen Behandelnden", enthaltend den "Austausch von Informationen entlang einer Behandlungskette» und die «integrierte Medikamentenverordnung», die «Prozesse zur dauerhaften Pflege eines Patientendossiers mit einem zusammenfassenden Auszug aus der Krankengeschichte (continuity of care)» sowie die «Administrativen Prozesse». In allen 6 Teilprojekten sollen die Grundlagen bis im Juli vorliegen, so dass im August die Entscheide gefällt werden können.

- Projekt Health Professional Card/HPC der FMH Die Ärztekammer hat im Dezember 2006 dem Zentralvorstand den Auftrag erteilt zur «Konzeption, Erstellung und Auslieferung einer HPC mit einem einfachen Zertifikat, der Erweiterungsmöglichkeit für ein qualifiziertes Zertifikat mit einer digitalen Signatur nach ZertES sowie der Sicherstellung der Interoperabilität mit der Versichertenkarte, sofern diese realisiert wird». Für die Laufzeit des Projektes wurden 3 Jahre vorgesehen (20072009), und es wurde ein Budget von 2,6 Mio. CHF bewilligt.

Im März 2009 wurde bei beiden Projektpartnern die Projektverantwortung neu geregelt. Auf Seiten Swisscom wurde das Projekt von den IT-Services an die Swisscom AG übertragen, bei der FMH übernahm M. Gauthey als ZV-Ressortleiterin eHealth die Projektleitung. Das bisherige Mandat mit dem externen Projektleiter wurde beendet. Die Projektgruppe wurde auf 5 Mitglieder reduziert. Im Moment stehen wir kurz vor dem Rollout der HPC, die ersten Karten werden im Juli ausgeliefert. Sämtliche FMH-Mitglieder werden zwischen Mai und Oktober 2009 (unaufgefordert) mit personalisierten Antragsunterlagen bedient. Die HPC wird zur Herstellung in Auftrag gegeben, sobald das unterschriebene Antragsformular mit der gewünschten Zertifikats-E-Mail-Adresse und einem aktuellen Passfoto bei der FMH eintrifft. Mit dem Rollout der HPC hat die FMH das Feld besetzt, die HPC muss bei der Versichertenkarte berücksichtigt werden, die FMH hat damit mehr Gewicht im Bereich der nationalen eHealth gewonnen, und es konnte ein wertvolles Netzwerk aufgebaut werden. Der neue Arzt- und FMH-Mitgliederausweis wird mit einem fortgeschrittenen (also nicht nur einfachen) Zerti- 
fikat ausgeliefert. Es ist die elektronische Identität des Arztes und identifiziert den Besitzer elektronisch sowohl als Person als auch als Arzt. Das Zertifikat kann zum elektronischen Signieren von Nachrichten und Dokumenten und zum Versenden und Empfangen vertraulicher Informationen (Ver-/Entschlüsseln) eingesetzt werden. Damit sich die Anwesenden eine Vorstellung vom Aussehen der HPC machen können, liegt auf jedem Platz eine mit der Vorderseite der HPC versehene «Coolcard» mit Pfefferminzbonbons.

- Ausblick

Die Arbeitsgruppe eHealth der FMH will weiterhin mit ihrer Arbeit zur Meinungsbildung und Integration innerhalb der FMH beitragen. Im Koordinationsorgan soll der Einbezug der Ärzteschaft verstärkt werden. Die auf nächstes Jahr vorgesehene Einführung der Versichertenkarte wird auf allen Ebenen eine grosse Herausforderung darstellen. Schliesslich soll die HPC in bestehende und geplante Anwendungen integriert werden, diverse Pilotversuche sind bereits angelaufen. Die Interoperabilität mit der Versichertenkarte ist sicherzustellen, und die Zusammenarbeit mit Apothekern und anderen Berufsgruppen soll gesucht bzw. gefördert werden.

Th. Keller/SZ erkundigt sich, weshalb auf der HPC der Titel fehlt. Der Präsident antwortet, dass einer unter anderen Gründen die eingeschränkte Feldlänge für den Namen ist. (Anm. der Protokollführerin: s. detaillierte Begründung auf www.fmh.ch, FAQ zur HPC.)

\section{SwissDRG: heutige Situation}

Der Präsident übergibt das Wort an Pierre-François Cuénoud für die Informationen aus seinem Ressort. In den vergangenen 6 Monaten hat sich der Verwaltungsrat hauptsächlich mit dem Gesuch an den Bundesrat befasst, das bis zum 30.6.2009 eingereicht werden muss, damit dieser 30 Monate Zeit zur Prüfung hat. Das Gesuch enthält an wichtigsten Punkten die Tarifstruktur und deren Anwendungsmodalitäten, das Kodierhandbuch mit dem Konzept zur Kodierrevision, die Instrumente und Mechanismen zur Gewährleistung der Qualität der Leistungserbringer sowie eine Schätzung über die Auswirkungen des Tarifs auf das Leistungsvolumen und die Kosten. Die Tätigkeiten der FMH in diesem Kontext bestehen einmal in der Einreichung von bis dato 1630 Anträgen an das Case Mix Office (CMO). Die meisten Anträge haben die Fachbereiche Orthopädie/Traumatologie, Allgemein- und Unfallchirurgie, Intensivmedizin, ORL, Urologie, Viszeralchirurgie sowie Gynäkologie und Geburtshilfe eingereicht. Am wenigsten Anträge stammen von der Anästhesie und Reanimation, der Handchirurgie, der Kinder- und Jungendpsychiatrie, Labormedizin und der Anthroposophischen Medizin. Alle Fachgesellschaften haben teilgenommen und Anträge eingereicht. $\mathrm{H}+$ hat einen Antrag eingereicht, alle übrigen Partner (GDK, sas, ZMT) keinen. Im laufenden Jahr findet eine neue Antragsrunde statt, die vom 1. Juli bis 20. September 2009 läuft. Wie im letzten Jahr wird es eine Unterstützung durch die FMH geben, die auf DRG-Spezialisten aus Deutschland und aus Schweizer Universitätsspitälern zurückgreifen kann. Der Antragsbedarf wurde in zwei Treffen in Bern (März) und Genf (April) erhoben. Es wird im Juni wiederum eine Reihe von Workshops mit den Fachgesellschaften geben. Geplant sind auch zwei verschiedene Seminare in Zusammenarbeit mit «H+ Bildung» unter den Titeln «SwissDRG: Grundlagen für Ärzte» und «Ärzte und Kodierung: Verantwortung unter SwissDRG». Diese Seminare werden im Laufe 2009 je dreimal durchgeführt. Wegen der sehr grossen Nachfrage ist eine Wiederholung im nächsten Jahr vorgesehen. Es wird auch über eine französische Ausgabe nachgedacht. Der Bereich SwissDRG innerhalb der FMH wurde personell verstärkt. Nach dem Weggang des Leiters Tarifdienst FMH übernimmt Beatrix Meyer ab 1. Juni die administrative Leitung des Tarifdienstes, neben der fachlichen Leitung des Bereiches SwissDRG. Hier stehen ihr neu Petra Ingenpass als stellvertretende Leiterin SwissDRG und als Assistentin Eva Naef zur Verfügung. Die fachliche Leitung für den Bereich Tarife und Verträge (umfassend TARMED und weitere ambulante Tarife) übernimmt $a b 1$. Juni Irène Marty. Weiter arbeiten in diesem Bereich mit: Thomas Kessler und Petra Meier.

Bei der Begleitforschung hat die FMH eine wesentlich umfassendere Sicht als ihre Partner, die der SwissDRG AG nur eine begleitende Beobachtung zuordnen wollen. Die FMH kann sich hier profilieren. Es gibt, wie an der heutigen Sitzung bereits erwähnt, eine Reihe von parlamentarischen Vorstössen (z. B. Bea Heim, Ignazio Cassis). Die Idee entstand, eine Ausschreibung für ein Konzept Begleitforschung SwissDRG zu machen, um hauptsächlich Antworten zu erhalten auf die Frage: Welche Themen und Parameter sind aus Sicht der FMH die wichtigsten? Und dann sekundär zu den Fragen: Wie misst man diese Parameter, wie muss die Datengrundlage sein, wer finanziert, wie setzt man das um ...? Der Auftrag wurde vergeben einerseits an das Institut Universitaire de médecine sociale et préventive (IUMSP, Lausanne, unter der Leitung von Prof. F. Paccaud), zum andern an Dr. Heinz Locher, Bern. In diesem Binom vereinigen sich medizinisches und ökonomisches Wissen. Die Arbeiten wurden mit einem Brainstorming gestartet.

Im Projekt SwissDRG verbleiben weiterhin einige Sorgen: Eine ganz grosse Sorge bleibt die Finanzierung der Weiterbildung. Das im Dezember 2007 beschlossene KVG schliesst eine Finanzierung von Forschung und universitärer Ausbildung aus, während wir davon ausgegangen sind, dass die Weiterbildung nicht zur universitären Ausbildung gehört. In den Verordnungen ist das zu unseren Ungunsten präzisiert: Die ganze Weiterbildung wird zur universitären Ausbildung geschlagen, obwohl wir wissen, dass zwei Drittel der Weiterbildung ausserhalb der Universitäten stattfinden. 
Eine andere Sorge bereitet die Tendenz, Arbeitsgruppen nebeneinander zu schalten. Das geschieht häufig dann, wenn die FMH in einer Arbeitsgruppe Dinge sagt, die von der andern nicht immer gern gehört werden. Dies wird vielfach damit umgangen, dass man eine parallele Arbeitsgruppe bildet, in welcher die FMH nicht vertreten ist. Eine weitere Sorge ist der Datenschutz, der von niemandem so richtig ernst genommen wird. Am Ende ist es aber der Arzt, der vor dem Richter steht und nicht der Spitaldirektor. Auch die Innovationen bereiten Sorge: Man kann keine gute Medizin machen, wenn man auf Innovationen verzichtet. Hier gab es den Versuch der Versicherer, das Thema auf später zu verschieben. Bleiben schliesslich die Qualitätskontrollen. Vieles wird gesagt, wenig umgesetzt, aber wir sind vom Gesetz her dazu verpflichtet. Es liegt also noch vieles auf dem Tisch. Uns bleibt nichts anderes als kämpferisch bleiben!

Ch. Bosshard/VSAO bedankt sich bei der FMH für das sehr positive Communiqué zur DRG-Petition, die auch vom VSAO unterstützt wird. Die Petition kann unter www.drg-petition.ch nachgelesen werden. Es geht genau um die von P.-F. Cuénoud angesprochenen Sorgenpunkte.

\section{Tarife und Verträge}

Ernst Gähler/ZV berichtet aus 4 Themenbereichen seines Ressorts.

\section{TARMED Suisse}

Im Leitungsgremium TARMED Suisse sind die vier Partner FMH, H+, santésuisse und MTK vertreten. Die Gesundheitsdirektorenkonferenz (GDK) nimmt als Beobachterin teil. Das Präsidium liegt im laufenden Jahr bei der FMH. TARMED Suisse ist zuständig für die Tarife. Die Organisation ist schwerfällig und langsam in den Entscheiden. Die Kommissionen PIK, PTK und PaKoDig sind der operative Teil. Zurzeit sind die strategische und die operative Ebene sehr vermischt. Deshalb hat der Ausschuss des Leitungsgremiums eine Entflechtung beschlossen, indem ab September niemand mehr aus den Kommissionen auch im Leitungsgremium sitzt. Das soll zu kürzeren Entscheidungswegen und schnelleren Entscheiden führen. Zudem soll der Geschäftsführer mehr eingebunden werden. Nicht ganz unentscheidend ist, dass die Partner zugestimmt haben, in der PTK eine medizinische Kommission zu bilden, welche alle medizinischen Fragen für die Kommission bearbeiten soll

Beim Projekt TARMED 2010 geht es um die Reorganisation des TARMED. Die FMH (DV und ZV) hat dem Projekt mit Bedingungen und Einschränkungen zugestimmt. H+ und MTK nehmen die gleiche Position ein. Das Problem ist, wie auch auf andern Ebenen, santésuisse, die kein sehr grosses Interesse hat, den TARMED zu reorganisieren, da dieser aus ihrer Sicht funktioniert und sie Angst hat vor einem allfälligen Kostenschub, zumal eine Kostensteigerung auch durch die Einführung der DRGs befürchtet wird. Santésuisse hat eine Kostensteuerungsvereinbarung ausgearbeitet, die noch vor Beginn des Projektstartes festhalten soll, wie die Kostenrealität dann auszusehen habe. FMH und $\mathrm{H}+$, und erstaunlicherweise auch die MTK, haben diese Vereinbarung abgelehnt. Die FMH will an LeiKov als tauglichem Instrument zur Kostensteuerung festhalten. Durch die Differenzen mit sas ist der Start des Projektes akut gefährdet.

Die FMH hat ein Projekt zur Revision des Dignitätskonzeptes initiiert, dem die Partner zugestimmt haben. Dabei geht es vor allem darum, die Frage der Besitzstände und der qualitativen Dignitäten neu zu regeln.

\section{Revision Analysenliste}

Diese «Baustelle» besteht seit 2006. Das BAG will kein separates Modell für das Praxislabor. BAG und santésuisse betrachten das Praxislabor als Nebengeschäft, und die Ärzteschaft darf an Nebengeschäften nichts verdienen. Das Praxislabor gehört aber zum Kerngeschäft des Praktikers. E. Gähler streift kurz die Geschichte des Point-of-Care-Tarifs. Ein juristisches Kurzgutachten hat festgestellt, dass es für dessen Einbringung in die Analysenliste keine Gesetzesänderung braucht, was vom BAG-Direktor erst kürzlich wieder in Abrede gestellt wurde. Nachdem die FMH während Monaten eine Dateneinsicht verlangte, wurde ihr diese am 20.1.2009 gewährt. Von 9 überprüften Positionen waren 7 fehlerhaft berechnet. Trotzdem hat Bundesrat Couchepin am 29.1.2009 beschlossen, die neue Analysenliste auf den 1. Juli 2009 einzuführen. Die FMH hat anschliessend alle möglichen politischen Register gezogen: Sehr gute Kontakte fanden statt mit den beiden Ständigen Kommissionen für soziale Sicherheit und Gesundheit (SGK) von Nationalrat und Ständerat (prominente Vertreter div. Parteien unterstützen die FMHPosition); Hearing mit der Geschäftsprüfungskommission des Nationalrats (sie kann nur Abläufe überprüfen, zum Inhalt aber keine Stellung nehmen). Die EAMGK (Eidg. Kommission für Analysen, Mittel und Gegenstände) hat am 7.4.2009 dem BAG einen Fragenkatalog mit 10 Punkten vorgelegt, weil der Vorsteher des EDI sie als beratende Kommission bei der letzten Version ganz einfach überging. Nach Intervention um eine erneute Dateneinsicht wurden E. Gähler und Roman Fried die Datengrundlagen (pdf-Dokumente und Excel-Tabellen!) zugestellt, mit dem Hinweis, die Accessdatenbank könne aus Gründen der Geheimhaltung nicht herausgegeben werden. Die FMH hat beim Eidg. Datenschutzbeauftragten wegen dieses Geheimhaltungsarguments ein Schlichtungsgesuch eingereicht. Ungereimtheiten gab es auch bei der Behauptung des BAG, die Laborkosten seien um $9 \%$ gestiegen, während die NewIndex-Zahlen klar aufzeigen, dass die Kosten seit 2006 bis heute nicht gestiegen sind. Eine erneute Dateneinsicht in die Accessdatenbank beim BAG zeigte, dass von 64 überprüften Grundversorgeranalysen, deren 25 plausibel, 39 aber nicht nachvollziehbar waren. All unsere Argumente nützen nichts, 
der BAG-Direktor hat bekräftigt, dass die neue Analysenliste am 1.7.2009 in der Version, wie sie am 29.1.2009 vorgestellt wurde, eingeführt wird.

Bleibt noch die Möglichkeit, den Point-of-CareTarif via santésuisse in die Analysenliste einzubringen. Die Verhandlungen sind jedoch harzig, und die aktuelle politische Situation macht das Ganze nicht einfacher.

Das BAG hat für das vorgesehene Monitoring eine Begleitgruppe eingesetzt, welcher Marc Müller/KHM und E. Gähler angehören. Für die Erarbeitung eines Konzeptes und die neutrale Beurteilung setzt das BAG eine Firma ein, deren Aufträge zu 90\% von der Bundesverwaltung kommen. Als Datenbasis war der Tarifpool von santésuisse vorgesehen, der sich aber dafür nicht eignet. Das BAG hat deshalb den Wunsch nach NewIndex-Zahlen geäussert. Die FMH wird ein eigenes Monitoring durchführen, ein Konzeptentwurf wurde erarbeitet, er wird mit der Arbeitsgruppe Labor nächste Woche diskutiert.

Abschliessend zeigt E. Gähler kurz auf, wie der weitere Aktionsplan der FMH aussieht.

Aus dem Plenum werden verschiedene Massnahmen vorgeschlagen, die unter dem Stichwort «ziviler Ungehorsam» subsumiert werden können. Marc Müller/KHM erinnert daran, dass das Argument gegen die neue Analysenliste war, die Qualität hochzuhalten. Der einzige Weg führt über ein sauberes Monitoring, das aufzeigt, dass das Praxislabor nicht mehr kostendeckend ist und damit der Anreiz, eine Praxis so auszurüsten, wie wir es brauchen, nicht mehr gegeben ist, was für die künftige medizinische Versorgung eine Katastrophe bedeutet.

\section{Taxpunktwertverhandlungen/ Taxpunktwertanpassung UV/IV/MV}

Die Taxpunktwertverhandlungen sind sowohl im KVG- wie im UVG-Bereich schwierig. Im KVG-Bereich bewegt sich wenigstens etwas: In einem Kanton kann der Taxpunktwert jetzt angehoben werden, was doch zeigt, dass die LeiKov gelebt wird.

Mit der MTK ist man seit eineinhalb Jahren im Gespräch. Eine Taxpunktwertanhebung um 4 Rappen konnte zwar erreicht werden, aber die MTK stellt ständig wechselnde Bedingungen. Die FMH hat konstruktiv mitgearbeitet und Lösungen vorgeschlagen, war aber bisher nicht erfolgreich. Unsere Geduld ist langsam zu Ende. Wenn die Antwort der MTK, auf die wir warten, negativ ist, dann werden der Delegiertenversammlung konkrete Kampfmassnahmen, die zurzeit in Vorbereitung sind, vorgeschlagen.

\section{Reduktion Vertriebsanteil DMA/SD}

Auch in diesem Bereich wird seit längerem von einer Reduktion des Vertriebsanteils um 3\% gesprochen. Für das BAG ist auch dies ein Nebengeschäft. Der Entscheid von Bundesrat Couchepin steht noch aus, jedoch ist im Zusammenhang mit der aktuellen Diskussion um die Senkung der Medikamentenpreise zu erwarten, dass der Vertriebsanteil reduziert wird. Der Bundesrat wird in Kürze beschliessen, gemunkelt wird über eine Senkung des Vertriebsanteils um 6\%, bei den Apothekern um $3 \%$. Über eine Motion von Verena Diener, die ein Splitting der Margen vorsieht (Ärzte sollen, weil sie weniger Umtriebe hätten, weniger Marge als die Apotheker erhalten). Es scheint auch, dass die Apotheker die LOA neu verhandelt hätten und einen sog. Polimedikationscheck einführen wollen, der mit 25 Taxpunkten (gehört hat man auch 45 Taxpunkte) abgegolten werden soll. Die Apotheker würden damit ihre Vertriebsanteilsenkung über die LOA wieder hereinholen. Selbstverständlich wird sich die FMH gegen ein Margensplitting zur Wehr setzen. Die Arbeitsgruppe DMA hat sich bereits Gedanken gemacht über mögliche Massnahmen.

E. Gähler zieht über alle Bereiche seines Ressorts das Fazit, dass nur der Weg über kleine Schritte möglich ist und man die Hoffnung hat, die Hartnäckigkeit habe präventive Wirkung auf andere Vorhaben des Bundes. Die Devise ist deshalb, konstruktiv und kämpferisch weiterzuarbeiten. Abschliessend bedankt er sich für die Unterstützung aus der Ärzteschaft und bei den Mitarbeitenden im Tarifdienst.

Peter Tschudi/ZH bewundert die Frustrationstoleranz von E. Gähler und fordert die Ärztekammer zu einem grossen Applaus für dessen grandiose Arbeit auf. E. Gähler bedankt sich dafür und bekräftigt, dass ihm dies Mut zum Weitermachen gibt.

V. Liguori/TI erkundigt sich, ob der Zentralvorstand die Möglichkeit habe, die Selbstdispensation auch in Kantonen zu verlangen, in denen es sie noch nicht gibt. Hp. Kuhn/Stv. Generalsekretär hält fest, dass das KVG den Kantonen die Kompetenz gibt, ob sie die SD zulassen und wie sie diese regeln wollen. Er sieht deshalb nicht, auf welcher Ebene die FMH hier Einfluss nehmen könnte.

Der Präsident übergibt Jean-Pierre Pavillon/VD das Wort zur Erläuterung seines Antrags Nr. 1 zu Traktandum 17.2. Für diesen stellt die ganze Laborproblematik nicht nur ein ökonomisches Problem für den frei praktizierenden Arzt dar, sondern man schadet damit auch dem Bild des Hausarztes, denn man nimmt ihm ein wesentliches Instrument zur Ausübung seiner ärztlichen Tätigkeit weg. Hinzu kommt die politische Tragweite: Die Mitglieder der SVM haben innert kürzester Zeit bei Patientenkontakten 40000 Unterschriften gesammelt, und bei Kontakten mit kantonalen und Bundesparlamentariern zeigten diese sehr viel Verständnis für die Position der Ärzteschaft. Eine breite Unterstützung ist da, hilft aber nicht weiter, weil die Analysenliste vom Chef des Departementes des Innern entschieden wird. Dessen Forderungen bezüglich anderer ärztlicher Tarife lassen befürchten, dass er in seiner Ermessensfreiheit auch diese nach Belieben wird manipulieren können. Wenn wir uns also jetzt nicht mit aller Kraft bei den Labortarifen wehren, werden wir das Gleiche beim TARMED und allen andern ärzt- 
lichen Tarifen erleben. Die Ärztegesellschaft des Kantons Waadt beantragt deshalb der Ärztekammer als oberstem Organ der FMH die Annahme einer Resolution zum Labor, wie sie im Entwurf unter dem Titel «Rettet die Medizin» vorliegt.

E. Gähler/ZV weist darauf hin, das die Resolution eigentlich nichts anderes fordert, als vom Ressort her auf verschiedensten Ebenen bereits angepackt wurde. Selbstverständlich kann man diese Resolution jetzt als FMH-Resolution beschliessen, aber man darf sich bezüglich ihrer Wirkung keine Illusionen machen. U. Stoffel/ZH zeigt sich besorgt, dass gewisse Formulierungen, die, provokativ ausgedrückt, bei unseren Partnern als eher «gewerkschaftliche Forderungen» daherkommen, uns den Zugang zu gewissen politischen Kommissionen, wo es um Lösungsfindungen geht, erschweren könnten.

Ph. Dreiding/ZH vermisst konkrete Kampfmassnahmen; Resolutionen zu beschliessen, scheint ihm in der aktuellen Situation nicht mehr der richtige Zeitpunkt zu sein.

Th. Keller/SZ teilt die Bedenken von U. Stoffel nicht ganz. Der Effekt könnte ebenso gut der sein, dass die politische Vertretung der Ärzteschaft nach aussen zeigt, dass sie durch klare Forderungen seitens der FMH-Mitglieder unter Druck steht. Er unterstützt deshalb die Resolution. F. Zogg/ZG schlägt einen Kompromiss vor, indem nur die erste Forderung des Resolutionsentwurfs als Resolution der Ärztekammer formuliert werden soll.

Nachdem das Wort nicht weiter verlangt wird, erläutert der Präsident das Abstimmungsprozedere:

Zunächst wird im Grundsatz darüber abgestimmt, ob eine Resolution gefasst werden soll. Anschliessend werden die Anträge J.-P. Pavillon/VD und F. Zogg/ZG einander gegenübergestellt.

Der Grundsatz, eine Resolution zu fassen, wird von den Delegierten mit überwältigender Mehrheit, bei 2 Gegenstimmen und 5 Enthaltungen, angenommen.

In der anschliessenden Gegenüberstellung der Anträge wird der Antrag von F. Zogg/ZG (verkürzte Version der SVM-Resolution) mit 92 Stimmen angenommen. Für den Antrag von J.-P. Pavillon/VD stimmen 49 Delegierte.

\section{Medical Education}

Kurzinformation über den Start des Schweizerischen Instituts für ärztliche Weiter- und Fortbildung (SIWF).

Der Präsident übergibt das Wort an Max Giger/ZV für dessen Bericht über das SIWF, dessen Bildung die ausserordentliche Ärztekammer im Dezember 2008 beschlossen hat. M. Giger weist einleitend darauf hin, dass auf der FMH-Website unter dem Bereich AWF/Verschiedenes der Jahresbericht 2008 der KWFB noch bis Ende Monat aufgeschaltet ist. Innerhalb der FMH ist das SIWF voll und ganz gegenüber der Ärztekammer rechenschaftspflichtig und hat deshalb auch ein eigenes Budget. Der Auftrag des SIWF ist eine effiziente
Berufsbildung unter Führung der Ärzteschaft. Die eine Aufgabe des SIWF ist die Durchführung der Weiterbildung im Auftrag des EDI gemäss Medizinalberufegesetz (MedBG), die andere Aufgabe ist die Fortbildung. Die ärztliche Berufsbildung soll sich voll und ganz nach dem Bedarf der Bevölkerung richten. Mit der demographischen Verschiebung wird die Zahl der älteren Personen über 65, ja über 80 Jahre, stark steigen, die chronischen Krankheiten werden beträchtlich zunehmen. Das SIWF hat dafür zu sorgen, dass die gesundheitliche Versorgung in der Schweiz weiterhin qualitativ und quantitativ hochstehend bleibt. Daraus ergeben sich folgende Aufgaben für das SIWF:

- Sicherstellen der «Workforce»: Die heute Weiterzubildenden sollen einmal in der Lage sein, die ältere Bevölkerung zu pflegen und zu versorgen.

- Stärkung der Kommunikation: Zuhören, Verständnis zeigen, Mitteilen einer Botschaft

- Verbesserung der Patientensicherheit

- Verbesserung des Schmerzmanagements

- Palliative Care: gemäss MedBG (Art. 18) muss die Ärzteschaft fähig sein, Patientinnen und Patienten bis zum Lebensende zu begleiten.

Es folgt ein Überblick über die heutige Weiterbildung in der Schweiz: 45 Facharzttitel, 27 Schwerpunkte, 28 Fähigkeitsausweise; die Weiterbildung erfolgt an rund 1200 Weiterbildungsstätten und in 650 Arztpraxen. 2008 wurden etwa 9000 Assistenzärztinnen und -ärzte weitergebildet. Am meisten Weiterbildungsstätten gibt es in der Inneren Medizin, am wenigsten (nur auf die grossen Fachgesellschaften bezogen) bei der Kinder- und Jugendmedizin. Weltweit ist eine Migration von Ärzten festzustellen aus Ländern mit tieferer Ärztedichte, mit etwas tieferem medizinischem Versorgungsstand in Länder mit höherem medizinischem Versorgungsstand. In Europa ist eine massive Migration von Nordosten nach Südwesten und eine allgemeine Migration von Nord nach Süd festzustellen. Die Schweiz ist ein bevorzugtes Land: Die Zahl der Schweizer Assistenzärzte nimmt kontinuierlich ab: 2008 waren weniger als $60 \%$ Schweizer, dafür wird eine stetige Zunahme von Assistenzärztinnen und -ärzten aus Deutschland verzeichnet. Die Schweiz muss immer mehr auf Länder ausserhalb von Europa zugreifen: In der Romandie sind es hauptsächlich nordafrikanische Länder, in der Deutschschweiz vermehrt Bulgarien (exportiert ca. $1 / 3$ der ausgebildeten Ärzte ins Ausland) und Rumänien.

Die Strukturen des SIWF umfassen eine Geschäftsleitung (4 Personen), den Vorstand (18 Personen und 11 Gäste) sowie die Plenarversammlung (57 Personen und 33 Gäste).

Vier Personen bilden die Geschäftsleitung: Max Giger, Präsident, zuständig für das Ressort Politik und Medizin; zwei Vizepräsidenten: Jean-Pierre Keller, Ressort Weiterbildungsstätten und Weiterbildungsstätten-Kommission; Hans Rudolf Koelz, Ressort Weiter- 
und Fortbildungsprogramme sowie Fortbildung, und schliesslich Christoph Hänggeli, zuständig für Administration, Personal und Recht.

Das Sekretariat des SIWF ist weiterhin bei der FMH in Bern ansässig und umfasst 18 Personen. Bereichsleiterinnen, alle seit vielen Jahren bei der FMH tätig, sind Petra Bucher-Baeriswyl (Allg. Sekretariat, SIWF Fortbildung - Website), Renate Jungo (Weiterbildungsstätten), Esther Würz (Titelkommission, Diplome) sowie Barbara Linder (Internationales); während vieler Jahre als Sekretärin/Sachbearbeiterin im Rechtsdienst tätig, hat sie letztes Jahr ihr berufsbegleitendes Jus-Studium mit dem Master of Law abgeschlossen. (Die Anwesenden würdigen dies mit Applaus.) Titelkommission (69 Fachdelegierte, 11 fachfremde Delegierte) und Weiterbildungsstättenkommission (60 Fachdelegierte, 11 fachfremde Delegierte) bestanden bereits in der KWFB und werden beibehalten.

An Projekten sind folgende aufzuführen: Akkreditierung 2011, Qualitätssicherung Weiterbildung (Assistentenumfrage mit der ETH und Visitationen); Assessmentprojekte mit dem Institut für Medizinische Lehre (IML) der Uni Bern (arbeitsplatzbasiertes Assessment; Webpool für Facharztprüfungen); Logbuch (in allen $43 \mathrm{WB}$ Programmen führen die Assistenten/-innen selbstverantwortlich ihr persönliches Logbuch, das sämtliche Aufzeichnungen über absolvierte Lerninhalte enthält); Finanzierung der Weiterbildung (eine politische Regelung muss bis Ende 2009 gefunden werden, man ist im Gespräch mit der GDK); Revision des Facharzttitels Innere Medizin (die Revision baut auf dem Lernzielkatalog der SMIFK auf; Ziel ist die Vereinigung Innere Medizin und Allgemeinmedizin, sie soll zu einer Stärkung der Hausarztmedizin führen); E-Plattform Fortbildung (Ziel ist die Qualitätssteigerung der Fortbildung; die Fortbildung soll über $m y \mathrm{FMH}$ individuell verwaltet werden können. und es soll möglich sein, sich sein eigenes Fortbildungsdiplom auszudrucken. Fernziel ist zudem, dass für die Registrierung an einer Fortbildungsveranstaltung und die Zuteilung der entsprechenden Credits die Health Professional Card/HPC eingesetzt werden kann). Schliesslich sollen im Bereich Fortbildung die SAMW-Richtlinien «Ärzteschaft und Industrie» umgesetzt werden, was konkret bedeutet, dass gewisse Fortbildungsveranstaltungen von der Industrie nicht mehr gesponsert werden dürfen.

Ch. Ramstein/VEDAG erinnert daran, dass die Amtszeit von Max Giger vor einem Jahr um zwei Jahre verlängert wurde, um damit die Möglichkeit zu schaffen, Ausschau nach einem Nachfolger als Leiter SIWF zu halten, der fachlich absolut kompetent ist und ein Pensum von geschätzt etwa $80 \%$ übernehmen muss. Der Votant macht darauf aufmerksam, dass mögliche Kandidaten für das Amt des Leiters SIWF heute wahrscheinlich eine Chefposition oder Ähnliches innehaben und deshalb mindestens ein halbes Jahr, wenn nicht ein Jahr zum Voraus ihr heutiges Arbeitspensum reduzieren oder ihre Stelle gar kündigen müssen. Da das Gerücht zirkuliert, dass im Dezember keine ausserordentliche ÄK stattfinden soll, der Leiter SIWF aber zwingend von der ÄK gewählt werden muss, ist Ch. Ramstein der Meinung, man müsste die Person heute bestätigen können.

M. Giger antwortet, dass der SIWF-Vorstand die Nachfolge intensiv besprochen, ein Profil erarbeitet und sich auf die Nachfolgesuche gemacht hat. Diskutiert wurde auch eine öffentliche Ausschreibung, die aber nicht möglich ist, weil der oder die Betroffene durch die ÄK gewählt werden muss und es sich gleichzeitig um eine politische wie eine Fachwahl handelt. Diskussionen mit einigen interessierten Kolleginnen und Kollegen sind am Laufen, mehr kann dazu im Moment nicht gesagt werden.

Die Wahl sollte sicher 6 Monate vor Amtsantritt stattfinden, so hat es der ZV an seiner letzten Sitzung festgehalten. M. Giger gibt seinem Vorredner Recht, dass der mögliche Kandidat oder die Kandidatin im Dezember als sog. President elect bestätigt werden müsste. Das Problem ist durch die erfolgte Ressortzuteilung an die beiden Vizepräsidenten etwas entschärft worden. Jacques de Haller präzisiert, dass der oder die Leiterin des SIWF gemäss Beschluss der a.o. Ärztekammer vom Dezember 2008 nicht mehr zwingend Mitglied des Zentralvorstandes sein muss, dass der ZV dies allerdings als höchst wünschenswert erachte.

Markus Furrer/Thorax-, Herz- und Gefässchirurgie macht darauf aufmerksam, dass die neuen EuQuMedRichtlinien viel schärfer geworden sind und weit über die schweizerischen Richtlinien hinaus gehen. Auch wenn wir also die Schweizer Richtlinien einhalten, werden Fortbildungsveranstaltungen nicht mehr bezahlt. So sind z.B. fast sämtliche FB-Kurse im Fachgebiet Chirurgie in Gefahr. Das wird alle FG in nächster Zeit ganz hart treffen, sollten die EuQuMed-Richtlinien so umgesetzt werden.

Ch. Ramstein/VEDAG kommt nochmals auf seine Frage bezüglich Zeitpunkt der Wahl des/der künftigen SIWF-Leiters/Leiterin zurück, auf die er noch keine klare Antwort erhalten hat. Der Präsident bekräftigt nochmals, dass der Prozess am Laufen ist und dass die Ärztekammer ganz klar wählen und nicht nur einen andernorts gefassten Entscheid «abwinken» wird.

\section{Varia}

Ignazio Cassis/ZV berichtet aus dem Nationalrat: Der Zulassungsstopp wurde mit grosser Mehrheit um weitere 2 Jahre verlängert. Er gilt neu nur noch für Spezialärzte (ausgeschlossen davon sind die Fachärzte Innere Medizin, die Pädiater, die Fachärzte Allgemeine Medizin sowie die Ärztinnen und Ärzte mit dem Titel Praktischer Arzt) mit Ausdehnung auf den ambulanten Spitalbereich. Die Kantone können explizit Bedingungen an eine Zulassung von Spezialisten stellen. Der Text geht nächste Woche in den Ständerat. Angesichts des Abstimmungsresultates glaubt I. Cassis nicht, dass der Ständerat bei seinen Divergenzen bleibt, so dass 
mit der Schlussabstimmung in 10 Tagen gerechnet werden kann. Ab 1. Januar 2010 und für die nächsten 2 Jahre wäre die neue Lösung in Kraft.

Franco E. Denti/TI erkundigt sich, ob für die Kantone etwas in Richtung Versorgungsplanung vorgesehen sei. I. Cassis/ZV antwortet, dass die Kantone nur die Kompetenz haben, gegenüber einem Individuum Bedingungen zu stellen. Allerdings könnte es sein, dass sie für den ambulanten Spitalbereich eine gewisse
Kompetenz für eine Bedarfsplanung erhalten könnten. Dazu muss aber die Schlussabstimmung abgewartet werden.

Nachdem das Wort nicht weiter verlangt wird, bedankt sich der Präsident für die gute Atmosphäre, in der die Verhandlungen stattfinden konnten, und schliesst die Sitzung der ordentlichen Ärztekammer um 17.15 Uhr.

\begin{tabular}{|c|c|c|c|}
\hline \multicolumn{4}{|l|}{ Glossar } \\
\hline \multirow[t]{2}{*}{ AHV } & \multirow{2}{*}{$\begin{array}{l}\text { Alters- und Hinterlassenenver- } \\
\text { sicherung }\end{array}$} & PTK & Paritätische Tarif-Kommission \\
\hline & & SAMW & Schweizerische Akademie \\
\hline AK & \multicolumn{2}{|l|}{ Ärztekammer } & der Medizinischen Wissenschaften \\
\hline AWF & Aus-, Weiter- und Fortbildung & sas & santésuisse \\
\hline BVG & Berufliches Vorsorge Gesetz & SÄZ & Schweizerische Ärztezeitung \\
\hline CMO & Casemix-Office & SD & Selbstdispensation \\
\hline DDQ & Daten, Demographie, Qualität & SFSM & Swiss Federation for Specialities \\
\hline DMA & \multicolumn{2}{|l|}{ Direkte Medikamentenabgabe } & in Medicine \\
\hline DRG & Diagnosis Related Groups & SGAI & Schweizerische Gesellschaft \\
\hline DV & \multicolumn{2}{|l|}{ Delegiertenversammlung } & für Allergologie und Immunologie \\
\hline \multirow{2}{*}{$\mathrm{fmCh}$} & Foederatio medicorum & SGGG & Schweiz. Gesellschaft für \\
\hline & chirurgicorum helvetica & & Gynäkologie und Geburtshilfe \\
\hline \multirow[t]{3}{*}{ GDK } & Schweiz. Konferenz der kantonalen & SGPP & Schweiz. Gesellschaft für \\
\hline & \multicolumn{2}{|l|}{ Gesundheitsdirektorinnen und } & Psychiatrie und Psychotherapie \\
\hline & -direktoren & SGH & Schweiz. Gesellschaft für \\
\hline GO & Geschäftsordnung & & Handchirurgie \\
\hline GPK & Geschäftsprüfungskommission & SGP & Schweiz. Gesellschaft für Pädiatrie \\
\hline КНM & Kollegium für Hausarztmedizin & SGTHGC & Schweizerische Gesellschaft für \\
\hline \multirow[t]{2}{*}{ КKA } & \multicolumn{2}{|l|}{ Konferenz der Kantonalen } & Thorax-, Herz- und Gefässchirurgie \\
\hline & Ärztegesellschaften & SIWF & Schweiz. Institut für Weiter- \\
\hline \multirow{2}{*}{$\begin{array}{l}\text { KVG } \\
\text { KVV }\end{array}$} & \multicolumn{2}{|l|}{ Krankenversicherungsgesetz } & und Fortbildung \\
\hline & $\begin{array}{l}\text { Krankenversicherungs- } \\
\text { verordnung }\end{array}$ & SMIFK & $\begin{array}{l}\text { Schweiz. Medizinische } \\
\text { Interfaktultätskommission }\end{array}$ \\
\hline KWFB & $\begin{array}{l}\text { Kommission für Weiter- } \\
\text { und Fortbildung }\end{array}$ & SMSR & $\begin{array}{l}\text { Société médicale } \\
\text { de la Suisse romande }\end{array}$ \\
\hline \multirow{2}{*}{$\begin{array}{l}\text { LOA } \\
\text { MedBG }\end{array}$} & Leistungsorientierte Abgabe & VEDAG & Verband deutschschweizerischer \\
\hline & \multicolumn{2}{|l|}{ Medizinalberufegesetz } & Ärztegesellschaften \\
\hline \multirow{2}{*}{$\begin{array}{l}\text { MTK } \\
\text { NAKO }\end{array}$} & \multirow{2}{*}{$\begin{array}{l}\text { Medizinaltarifkommission } \\
\text { Nationale Konsolidierungsstelle }\end{array}$} & VLSS & Verein der Leitenden Spitalärzte \\
\hline & & & der Schweiz \\
\hline OBSAN & $\begin{array}{l}\text { Schweizerisches Gesundheits- } \\
\text { observatorium }\end{array}$ & VSAO & $\begin{array}{l}\text { Verband Schweizerischer } \\
\text { Assistenz- und Oberärztinnen }\end{array}$ \\
\hline ORL & Oto-Rhino-Lanryngologie & & und -ärzte \\
\hline \multirow[t]{2}{*}{ PaKoDig } & \multirow{2}{*}{$\begin{array}{l}\text { Paritätische Kommission Dignitäten } \\
\text { und Sparten }\end{array}$} & ZMT & Zentralstelle für Medizinal-Tarife \\
\hline & & ZV & Zentralvorstand \\
\hline PIK & $\begin{array}{l}\text { Paritätische Interpretations- } \\
\text { kommission }\end{array}$ & & \\
\hline
\end{tabular}

